\title{
Proper-time evaluation of the effective action: Unequal masses in the loop
}

\author{
A. A. Osipov๑* \\ Joint Institute for Nuclear Research, Bogoliubov Laboratory of Theoretical Physics, \\ 141980 Dubna, Russia
}

(Received 11 October 2021; accepted 21 October 2021; published 29 November 2021)

\begin{abstract}
The Fock-Schwinger proper-time method is used to derive the effective action in the field theory with the chiral $U(3) \times U(3)$ symmetry explicitly broken by unequal masses of heavy particles. The one-loop effective action is presented as a series in inverse powers of heavy masses. The first two Seeley-DeWitt coefficients of this expansion are explicitly calculated. This powerful technique opens a promising avenue for studying explicit flavor symmetry-breaking effects in the effective field theories.
\end{abstract}

DOI: 10.1103/PhysRevD.104.105019

\section{INTRODUCTION}

The heat kernel technique [1] was introduced to quantum theory in works of Fock [2,3] and later pushed forward by Nambu [4] and Schwinger [5]. In combination with the background field method, this allowed DeWitt to develop the manifestly covariant approach to gauge field theory [6], and quantum gravity [7]. The method allows the essentially nonperturbative and nonlocal extensions [8-11]. It has been widely used in QCD to construct effective meson Lagrangians [12,13], in chiral gauge theories to study chiral anomalies [14], in cosmology to calculate geometric entropy [15], in QED to find Casimir energies and forces [16], etc.

In all above-mentioned cases and in many others [17], it becomes necessary to calculate the determinant of a positive-definite elliptic operator that describes quadratic fluctuations of quantum fields in the presence of some external or background fields and which in a compact form contains all the information about the one-loop contribution of quantum fields. The result is an asymptotic expansion for the effective action of the theory in powers of the proper time with Seeley-DeWitt coefficients $a_{n}(x, y)$. These coefficients are polynomials in the background fields and describe, in the coincidence limit $y \rightarrow x$, the local vertices of the corresponding effective Lagrangian. It is remarkable that each term of the expansion is invariant under the action of the group of the internal symmetry, if the theory possesses this symmetry. This follows from the general covariance of the formalism.

\footnotetext{
*aaosipov@jinr.ru
}

Published by the American Physical Society under the terms of the Creative Commons Attribution 4.0 International license. Further distribution of this work must maintain attribution to the author(s) and the published article's title, journal citation, and DOI. Funded by SCOAP.
When quantum fields have large equal masses $m$, it is easy to resort to an expansion in the inverse powers of mass, which is valid when all background fields and their derivatives are small compared to the mass of the quantum field. In this case, the asymptotic coefficients $a_{n}$ do not change. Such long wavelengths $(\lambda \gg 1 / m)$ expansion allows one to obtain an action that takes into account effectively the leading low-energy effect of virtual heavy states. This scenario is realized in theories with spontaneously broken symmetry, or in the theories with a large bare mass. A typical example of the first type is the Nambu-Jona-Lasinio (NJL) model [18,19], where ground state in the strong-coupling regime is found to be separated by a gap from the excited states (quasiparticles), which are identified with nucleons. Reinterpreting nucleons as quarks, one obtains a low-energy meson action from one-loop quark dynamics [20]. The proper-time method is especially useful here $[12,13]$. Examples of the second type arise under extension of some effective field theory $X$ with symmetry group $G$ to the other effective theory $X^{\prime}$, when $X^{\prime}$ contains heavy degrees of freedom belonging to some representation of $G$. At present, such theories are being actively studied in the context of extending the standard model of electroweak interactions [21].

In realistic models one is often confronted with the difficulty that the flavor symmetry is broken by large unequal masses $M=\operatorname{diag}\left(m_{1}, m_{2}, \ldots, m_{f}\right)$. In this case the complete factorization of $M$ in the heat kernel is impossible because $M$ does not commute with the rest of an elliptic operator. The consequence is that the Seeley-DeWitt coefficients depend in a complex way on both the fields and the mass-dependent constants of their interactions. The treatment of such case is known to be an onerous task, especially when both Bose and gauge fields are present in the elliptic operator [22-24].

Recently [25,26], a new algorithm based on the propertime method has been proposed for deriving the effective 
action in a theory with heavy virtual fermions (or bosons) of unequal masses belonging to some representation of the symmetry group $G$. These short papers contain the main idea and the final result. Nontrivial calculation details have been omitted due to their complexity. The purpose of the present contribution is to supply all necessary details of such nontrivial calculations, without which it is difficult to be convinced of the validity of the previously stated results.

The new algorithm generalizes the standard large mass expansion of the heat kernel to the case of unequal masses by the formula

$$
e^{-t\left(M^{2}+A\right)}=e^{-t M^{2}}\left[1+\sum_{n=1}^{\infty}(-1)^{n} f_{n}(t, A)\right]
$$

where $M=\operatorname{diag}\left(m_{1}, m_{2}, \ldots, m_{f}\right)$ is a diagonal mass matrix; $t$ is the proper-time parameter; the expression in the square brackets is the time-ordered exponential $\mathrm{OE}[-A](t)$ of $A(s)=e^{s M^{2}} A e^{-s M^{2}}$, and $A$ is a positivedefinite self-adjoint elliptic operator in some background (its explicit form will be clarified later), accordingly

$f_{n}(t, A)=\int_{0}^{t} d s_{1} \int_{0}^{s_{1}} d s_{2} \ldots \int_{0}^{s_{n-1}} d s_{n} A\left(s_{1}\right) A\left(s_{2}\right) \ldots A\left(s_{n}\right)$.

If masses are equal, this formula yields the well-known large mass expansion with standard Seeley-DeWitt coefficients $a_{n}(x, y)$ [14]. In fact, formula (1) is an extension of the Schwinger method used to isolate the divergent aspects of a calculation in integrals with respect to the proper time $[5,7]$ to the noncommutative algebra.

There is a simple heuristic argument that explains why this formula is also relevant for describing the generalized $1 / M$ series. Indeed, the $1 / M$ expansion is known to be valid when all background fields and their derivatives are small compared to the mass of quantum fields. Therefore, factoring $e^{-t M^{2}}$ one separates the leading contribution. The remaining part of the heat kernel may be unambiguously evaluated by expanding it in a power series in $t$ about $t=0$. As a consequence, the Seeley-DeWitt coefficients $a_{n}$ receive corrections: $a_{n} \rightarrow b_{n}=a_{n}+\Delta a_{n}$, where $\Delta a_{n}$ vanish in the limit of equal masses.

Currently, there are two methods for deriving quantum corrections induced by virtual states of unequal masses. In [22-24], the heat kernel is evaluated on the basis of the modified DeWitt Wentzel-Kramers-Brillouin (WKB) form. This yields a different asymptotic series for the right-hand side of Eq. (1), and, consequently, the different expressions for $\Delta a_{n}$. The approach proposed in [27-29] starts from the formula (1), but afterwards an additional resummation of the asymptotic series is applied. This essentially simplifies the calculations, but changes the structure of the $1 / M$ series. As a result, one loses correspondence between a mass-dependent factor at the effective vertex and a flavor content of the oneloop Feynman diagram which generates the vertex. Here I abandon this resummation.

The utility of the proper-time technique is that it reduces the task of the large mass expansion to a simple algebraic problem which requires less work than one needs for the corresponding Feynman diagrams calculation in momentum space. In the following, we consider a quite nontrivial case of the chiral $U(3) \times U(3)$ symmetry broken by the diagonal mass matrix $M=\operatorname{diag}\left(m_{1}, m_{2}, m_{3}\right)$ to demonstrate the power of the method. To find the two leading contributions $b_{1}(x, x)$ and $b_{2}(x, x)$ in the $1 / M$ expansion of the effective action, one requires to consider only four terms of the series (1) that results in more than a hundred effective vertices.

The effects of flavor symmetry breaking are currently important in many physical applications: in studies of physics beyond standard model to construct the low-energy effective action by integrating out the heavy degrees of freedom [21,30]; in two Higgs doublet models [31] to address the problem of almost degenerate Higgs states at $125 \mathrm{GeV}[32,33]$; in the low-energy QCD to study the $S U(3)$ and isospin symmetry-breaking effects [34]. These effects are known to be very important to explore the QCD phase diagram [35], to study a formation of the strangequark matter [36,37], to study nuclear matter in extreme conditions that arose in nature at the early stages of the evolution of the Universe and in the depths of neutron stars $[38,39]$. The method described below, in particular, can be used for obtaining low-energy meson effective Lagrangian in the framework of the NJL model, as an alternative to the approaches developed in [13,40].

The paper is organized as follows. In Sec. II, we formulate the method and present the basic steps required to construct the desired 1/ $M$ expansion. Section III sets out the necessary details related to the calculation of the first two coefficients of the asymptotic series. In Sec. IV, we present the effective Lagrangian describing the self-energy and interactions of zero-spin and one-spin bosons in Minkowski space-time induced by the intermediate oneloop quark diagrams. A short summary and some concluding remarks are given in Sec. V. Many important technical points related to our calculations are collected in six appendixes.

\section{PROPER-TIME EXPANSION}

In this section we obtain the $1 / M$ series of the effective one-loop action in Euclidian space and explicitly single out the structures necessary for calculating the first two coefficients of this asymptotic expansion.

\section{A. Determinant of the Dirac operator}

The logarithm of the formal determinant of the selfadjoint elliptic operator of the second order describes the 
one-loop radiative corrections to classical theory. In the following, we are interested in the real part of the effective action resulting from the calculation of the determinant of the Dirac operator $D$ in the background scalar $s$, pseudoscalar $p$, vector $v_{\mu}$, and axial vector $a_{\mu}$ fields. The propertime method cannot be applied directly to fermions, since the Dirac operator $D$ is a first-order elliptic operator, and its spectrum is unbounded both above and below. Instead, one should consider the functional

$$
W_{E}=\ln \left|\operatorname{det} D_{E}\right|=-\int_{0}^{\infty} \frac{d t}{2 t} \rho_{t, \Lambda} \operatorname{Tr}\left(e^{-t D_{E}^{\dagger} D_{E}}\right),
$$

representing a real part of the one-loop effective action in Euclidean space as the integral over the proper-time $t$. Notice that the Hermitian operator $D_{E}^{\dagger} D_{E}$ is a second-order elliptic operator, unbounded above, so we can use the proper-time method to regularize it precisely as for bosons. The integral diverges at the lower limit; therefore, a regulator $\rho_{t, \Lambda}$ is introduced, where $\Lambda$ is an ultraviolet cutoff. Since we will carry out calculations in Euclidean space (the subscript $E$ marks this), and the obtained result will be analytically continued to Minkowski space, we will adhere to certain rules of transition from one space to another, which we collect in Appendix A.

For definiteness, suppose that one is dealing with the effective action arising due to integration over light-quark degrees of freedom. In this case, the Dirac operator $D_{E}$ in Euclidean four-dimensional space has the form

$$
D_{E}=i \gamma_{\alpha} d_{\alpha}-M+s+i \gamma_{5 E} p,
$$

where $d_{\alpha}=\partial_{\alpha}+i \Gamma_{\alpha}, \Gamma_{\alpha}=v_{\alpha}+\gamma_{5 E} a_{\alpha}, \alpha=1,2,3,4$. The external fields are embedded in the flavor space through the set of matrices $\lambda_{a}=\left(\lambda_{0}, \lambda_{i}\right)$, where $\lambda_{0}=\sqrt{2 / 3}$ and $\lambda_{i}$ are the eight $S U(3)$ Gell-Mann matrices; for instance, $s=s_{a} \lambda_{a}$, and so on for all fields. The quark masses are given by the diagonal matrix $M=\operatorname{diag}\left(m_{1}, m_{2}, m_{3}\right)$ in the flavor space. The symbol " $T r$ " denotes the trace over Dirac $(D) \gamma$ matrices, color $(c) S U(3)$ matrices, and flavor $(f)$ matrices, as well as integration over coordinates of the Euclidean space: $\operatorname{Tr} \equiv \operatorname{tr}_{I} \int d^{4} x_{E}$, where $I=(D, c, f)$. The trace in the color space is trivial: it leads to the overall factor $N_{c}=3$. The dependence on external fields in $D_{E}$ after switching to the Hermitian operator

$$
D_{E}^{\dagger} D_{E}=M^{2}-d^{2}+Y
$$

is collected in $Y$ and the covariant derivative $d_{\alpha}$. In the following we do not need an explicit expression for $Y$; nevertheless, for completeness, we include this expression in Appendix A.

If quarks were massless, the theory would have a global $U(3)_{L} \times U(3)_{R}$ chiral symmetry. It is known, however, that the ground state of QCD is not invariant under the action of chiral group. As a result, the entire system undergoes a phase transition accompanied by the appearance of a gap in the fermion spectrum. Quarks get their equal nonzero constituent masses.

Additionally, due to explicit breaking of chiral symmetry, realized through the mass terms of current quarks, one can easily find that the inequality of current masses after spontaneous breaking of chiral symmetry leads to the inequality of constituent quark masses.

Thus, we arrive at a problem in which one needs to study the properties of a system at large scales, i.e., one needs an expansion of the effective action in the inverse powers of large unequal masses of quarks:

$$
M=\sum_{i=1}^{3} m_{i} E_{i}, \quad\left(E_{i}\right)_{j k}=\delta_{i j} \delta_{i k}, \quad E_{i} E_{j}=\delta_{i j} E_{j} .
$$

The matrix $E_{i}$ maps the point $(u, d, s)$ of the threedimensional flavor space to the point $(u, 0,0)$ if $i=1$, to the point $(0, d, 0)$ if $i=2$, and to the point $(0,0, s)$ if $i=3$. Thus $E_{i}$ is an orthogonal projection onto the flavor space which can be expressed through the $\lambda$ matrices:

$$
\begin{gathered}
E_{1} \equiv \lambda_{u}=\frac{1}{\sqrt{6}} \lambda_{0}+\frac{1}{2} \lambda_{3}+\frac{1}{2 \sqrt{3}} \lambda_{8}, \\
E_{2} \equiv \lambda_{d}=\frac{1}{\sqrt{6}} \lambda_{0}-\frac{1}{2} \lambda_{3}+\frac{1}{2 \sqrt{3}} \lambda_{8}, \\
E_{3} \equiv \lambda_{s}=\frac{1}{\sqrt{6}} \lambda_{0}-\frac{1}{\sqrt{3}} \lambda_{8} .
\end{gathered}
$$

Notice also the following useful relations which are important for our calculations. The first formula is a direct consequence of the fact that the quark mass is given by the diagonal matrix

$$
e^{-t M^{2}}=\sum_{i=1}^{3} e^{-t m_{i}^{2}} E_{i}
$$

The second formula reflects the projection property of the $E_{i}$ matrices:

$$
E_{i} A E_{j}=A_{i j} E_{i j}
$$

where $\left(E_{i j}\right)_{m n}=\delta_{i m} \delta_{j n}$, in particular, $E_{i i}=E_{i}$. It is true for any flavor matrix $A$ whose entries are given by $A_{i j}$. Note that here and in the following we sum over repeated flavor indices only when a symbol of the sum is explicitly written out. The orthogonal basis $E_{i j}$ has the properties 


$$
\begin{aligned}
\operatorname{tr}_{f}\left(E_{i j}\right) & =\delta_{i j}, \\
\operatorname{tr}_{f}\left(E_{i j} E_{k l}\right) & =\delta_{i l} \delta_{j k}, \\
\operatorname{tr}_{f}\left(E_{i j} E_{k l} E_{m n}\right) & =\delta_{i n} \delta_{j k} \delta_{l m}, \\
\ldots \ldots \ldots \ldots \ldots & \ldots \ldots \ldots \ldots \\
\operatorname{tr}_{f}\left(E_{i_{1} i_{2}} \ldots E_{i_{2 n-1} i_{2 n}}\right) & =\delta_{i_{1} i_{2 n}} \delta_{i_{2} i_{3}} \ldots \delta_{i_{2 n-2} i_{2 n-1}} .
\end{aligned}
$$

To advance in the evaluation of expression (3), we use the Schwinger technique of a fictitious Hilbert space [5]. Then, a matrix element of a quantum-mechanical operator can be treated as

$$
\operatorname{Tr}\left(e^{-t D_{E}^{\dagger} D_{E}}\right) \equiv \int d^{4} x_{E} \operatorname{tr}_{I}\left\langle x\left|e^{-t D_{E}^{\dagger} D_{E}}\right| x\right\rangle .
$$

The use of a plane wave with Euclidean 4-momenta $k$, $\langle x \mid k\rangle$, as a basis greatly simplifies the calculations (details are given in Appendix B) and leads to the representation of the effective action as an integral over the fourmomentum $k_{\alpha}$ :

$W_{E}=-\int d^{4} x \int \frac{d^{4} k}{(2 \pi)^{4}} e^{-k^{2}} \int_{0}^{\infty} \frac{d t}{2 t^{3}} \rho_{t, \Lambda} \operatorname{tr}_{I}\left[e^{-t\left(M^{2}+A\right)}\right]$,

where

$$
A=-d^{2}-2 i k d / \sqrt{t}+Y
$$

is a self-adjoint operator in Hilbert space, and the summation over four-vector indices in (15) are implicit.

\section{B. The case of equal masses}

Before proceeding with our calculations, it is appropriate to discuss the simplest case of the large- $M$ expansion, i.e., the case when the mass matrix $M$ is $M=\operatorname{diag}(m, m, m)$; then $[M, A]=0$ and we have

$$
e^{-t\left(M^{2}+A\right)}=e^{-t M^{2}} e^{-t A}=e^{-t m^{2}} \sum_{n=0}^{\infty} t^{n} a_{n}(x, x) .
$$

Here $a_{n}(x, x)$ are the Seeley-DeWitt coefficients $a_{n}(x, y)$ in a coincidence limit $x=y$, which depend on the background fields and their derivatives, except $a_{0}(x, x)=1$. Integration over four-momentum and proper time in (14) is straightforward and we obtain a well-known result,

$$
W_{E}=-\int \frac{d^{4} x}{32 \pi^{2}} \sum_{n=0}^{\infty} J_{n-1}\left(m^{2}\right) \operatorname{tr}_{I} a_{n}(x, x),
$$

where the proper-time integrals $J_{n}\left(m^{2}\right)$ are

$$
J_{n}\left(m^{2}\right)=\int_{0}^{\infty} \frac{d t}{t^{2-n}} e^{-t m^{2}} \rho_{t, \Lambda}
$$

In the case of two subtractions $\rho_{t, \Lambda}=1-\left(1+t \Lambda^{2}\right) e^{-t \Lambda^{2}}$ at the large scale $\Lambda$, one finds from (18)

$$
\begin{gathered}
J_{0}\left(m^{2}\right)=\Lambda^{2}-m^{2} \ln \left(1+\frac{\Lambda^{2}}{m^{2}}\right), \\
J_{1}\left(m^{2}\right)=\ln \left(1+\frac{\Lambda^{2}}{m^{2}}\right)-\frac{\Lambda^{2}}{\Lambda^{2}+m^{2}} .
\end{gathered}
$$

The choice of the regularization is closely related to the specific problem under study. Various examples of the proper-time regularization can be found in [14]. The PauliVillars regularization we used is usually applied in the NJL model, where the cutoff $\Lambda$ is a scale of spontaneous chiral symmetry breaking.

The functions $J_{n}\left(m^{2}\right)$, for $n>1$, as $m^{2}$ becomes very large are asymptotically equivalent to $m^{-2(n-1)}$, that is, the expansion (17) is in inverse powers of $m^{2}$. For the series to converge, it is necessary not only that the mass $m$ be large, but also that the background fields change slowly over distances of the order of the fermion field Compton wavelength $1 / \mathrm{m}$. If these criteria are not met, then the production of real quark-antiquark pairs becomes essential, and the expansion is not suitable for applications.

\section{The case of unequal masses}

Let us return now to the formula (14) and show how one can extend the above tool to the case $[M, A] \neq 0$. To make progress in our calculations, we use the formula (1) allowing to factorize the exponent with a noncommuting diagonal mass matrix $M$. Under a flavor trace, it yields, for instance,

$$
\operatorname{tr}_{f}\left(e^{-t M^{2}}\right)=\sum_{i=1}^{3} e^{-t m_{i}^{2}} \operatorname{tr}_{f} E_{i}=\sum_{i=1}^{3} e^{-t m_{i}^{2}}
$$

The result of calculations for the remaining terms can be represented by the formula

$$
\begin{aligned}
\operatorname{tr}_{f}\left(e^{-t M^{2}} f_{n}(t, A)\right) \\
=\frac{t^{n}}{n !} \sum_{i_{1}, i_{2}, \ldots, i_{n}}^{N_{f}} c_{i_{1} i_{2} \ldots i_{n}}(t) \operatorname{tr}_{f}\left(A_{i_{1}} A_{i_{2}} \ldots A_{i_{n}}\right),
\end{aligned}
$$

where $n \geq 1$ and the notation $A_{i} \equiv E_{i} A$ is used. The coefficients $c_{i_{1} i_{2} \ldots i_{n}}(t)$ are totally symmetric with respect to any permutation of indices and are easily calculated. The necessary details of such calculations and useful properties of coefficients are collected in Appendix C.

To ensure the fundamental cyclic property of the trace $\operatorname{tr}(A B)=\operatorname{tr}(B A)$, we define 


$$
\begin{aligned}
\operatorname{tr}_{f}\left(A_{i_{1}} A_{i_{2}} \ldots A_{i_{n}}\right) & =\frac{1}{n} \sum_{\text {cycl perm }} A_{i_{1} i_{2}} A_{i_{2} i_{3}} \ldots A_{i_{n} i_{1}} \\
& \equiv\left\langle A_{i_{1} i_{2}} A_{i_{2} i_{3}} \ldots A_{i_{n} i_{1}}\right\rangle .
\end{aligned}
$$

Here we used Eqs. (11) and (12) to calculate the trace. The sum over a cyclic permutation of $A_{i j}$ adds nothing to the standard definition of a trace if $A$ is a matrix. However, in the case when $A$ contains open derivatives, i.e., is a differential operator, cyclic permutation in the trace may change the result. This is why it is necessary to ensure the guaranteed fulfillment of this fundamental property of the trace, which we do with the formula (23).

It should be emphasized that here we will restrict ourselves to considering only those terms that survive in the limit $M \rightarrow \infty$. In the case of equal masses, this approximation corresponds to considering heat coefficients $a_{1}$ and $a_{2}$. To isolate such a contribution, it is necessary to limit ourselves to the terms of order $t^{2}$ at most. It means that at the level of $A_{i}$-dependent expressions one should expand up to and including the $t^{4}$ order, since $A_{i}$ has the term $\propto 1 / \sqrt{t}$,

$$
\begin{aligned}
\operatorname{tr}_{f}\left(e^{-t\left(M^{2}+A\right)}\right)= & \sum_{i=1}^{3} c_{i}(t)-t \sum_{i=1}^{3} c_{i}(t) \operatorname{tr}_{f} A_{i} \\
& +\frac{t^{2}}{2 !} \sum_{i, j} c_{i j}(t) \operatorname{tr}_{f}\left(A_{i} A_{j}\right) \\
& -\frac{t^{3}}{3 !} \sum_{i, j, k} c_{i j k}(t) \operatorname{tr}_{f}\left(A_{i} A_{j} A_{k}\right) \\
& +\frac{t^{4}}{4 !} \sum_{i, j, k, l} c_{i j k l}(t) \operatorname{tr}_{f}\left(A_{i} A_{j} A_{k} A_{l}\right)+\mathcal{O}\left(t^{5}\right) .
\end{aligned}
$$

Note that the series is of the mixed type, i.e., it has also exponents depending on $t$, hidden in the coefficients $c_{i_{1} i_{2} \ldots i_{n}}(t)$. In the limit $m_{1}=m_{2}=m_{3}=m$, these coefficients shrink to $c(t)=\exp \left(-t m^{2}\right)$ and the massdependent exponent is totally factorized. This way one can recover the standard inverse mass expansion.

As it will be shown below, the term with the coefficient $c_{i_{1} i_{2} \ldots i_{n}}$ corresponds to the contact contribution of the Feynman diagram with $n$ internal quark lines. It means that $c_{i}$ can be associated with the tadpole contribution, $c_{i j}$ with the self-energy part, and $c_{i j k}$ and $c_{i j k l}$ with the triangle and box contributions. The indices contain also information on the flavor content of such one-loop diagrams. For instance, $c_{123}(t)$ corresponds to the triangle originated by propagators of up, down, and strange constituent quarks.

Now, one can substitute $A_{i}=E_{i} A$ by its expression (15) and integrate over four-momentum $k_{\alpha}$ in (14) by using the formula

$$
\int \frac{d^{4} k}{(2 \pi)^{4}} e^{-k^{2}} k_{\alpha_{1}} k_{\alpha_{2}} \ldots k_{\alpha_{2 n}}=\frac{\delta_{\alpha_{1} \alpha_{2} \ldots \alpha_{2 n}}}{(4 \pi)^{2} 2^{n}}
$$

It is evident that the corresponding integral of an odd number of four-momentum $k_{\alpha}$ is zero. Totally symmetric tensor $\delta_{\alpha_{1} \alpha_{2} \ldots \alpha_{2 n}}$ is determined by the recurrent relation

$$
\delta_{\alpha_{1} \alpha_{2} \ldots \alpha_{2 n}}=\sum_{i=2}^{2 n} \delta_{\alpha_{1}}^{\alpha_{i}} \delta_{\alpha_{2} \ldots \alpha_{i-1} \alpha_{i+1} \ldots \alpha_{2 n}}
$$

see also Eq. (B8) for details. This yields

$$
\begin{aligned}
\int \frac{d^{4} k}{(2 \pi)^{4}} e^{-k^{2}} \operatorname{tr}_{f}\left(e^{-t\left(M^{2}+A\right)}\right) \\
=\frac{1}{(4 \pi)^{2}}\left\{\sum_{i=1}^{3} c_{i}(t)-t \sum_{i=1}^{3} c_{i}(t) \operatorname{tr}_{f}\left[E_{i}\left(Y-d^{2}\right)\right]-t \sum_{i, j} c_{i j}(t) \operatorname{tr}_{f}\left(E_{i} d_{\alpha} E_{j} d_{\alpha}\right)+\frac{t^{2}}{2} \sum_{i, j} c_{i j}(t) \operatorname{tr}_{f}\left[E_{i}\left(Y-d^{2}\right) E_{j}\left(Y-d^{2}\right)\right]\right. \\
\quad+\frac{t^{2}}{3} \sum_{i, j, k} c_{i j k}(t) \operatorname{tr}_{f}\left[E_{i} d_{\alpha} E_{j} d_{\alpha} E_{k}\left(Y-d^{2}\right)+E_{i} d_{\alpha} E_{j}\left(Y-d^{2}\right) E_{k} d_{\alpha}+E_{i}\left(Y-d^{2}\right) E_{j} d_{\alpha} E_{k} d_{\alpha}\right] \\
\left.\quad+\frac{t^{2}}{3 !} \sum_{i, j, k, l} c_{i j k l}(t) \delta_{\alpha \beta \gamma \delta} \operatorname{tr}_{f}\left(E_{i} d_{\alpha} E_{j} d_{\beta} E_{k} d_{\gamma} E_{l} d_{\delta}\right)\right\}+\mathcal{O}\left(t^{3}\right) .
\end{aligned}
$$

We put this result into (14) and calculate the integrals over the proper time. Integration turns the coefficients $c_{i_{1} i_{2} \ldots i_{n}}(t)$ into functions $J_{i_{1} i_{2} \ldots i_{n}}$ depending on the masses of the fermion fields and the cutoff $\Lambda$. These functions describe the leading contributions in the $1 / M^{2}$ expansion of the corresponding one-loop Feynman diagrams, to be precise, their local parts which dominate in the limit $M \rightarrow \infty$. Here we present the result of such calculations. All necessary details can be found in Appendix D. 


$$
\begin{aligned}
W_{E}= & -\frac{N_{c}}{32 \pi^{2}} \int d^{4} x\left\{-\sum_{i} J_{0}\left(m_{i}^{2}\right) \operatorname{tr}_{D f}\left[E_{i}\left(Y-d^{2}\right)\right]-\sum_{i, j} J_{0}\left(m_{i}^{2}, m_{j}^{2}\right) \operatorname{tr}_{D f}\left(E_{i} d_{\alpha} E_{j} d_{\alpha}\right)\right. \\
& +\frac{1}{2} \sum_{i, j} J_{i j} \operatorname{tr}_{D f}\left[E_{i}\left(Y-d^{2}\right) E_{j}\left(Y-d^{2}\right)\right]+\frac{1}{3} \sum_{i, j, k} J_{i j k} \operatorname{tr}_{D f}\left[E_{i} d_{\alpha} E_{j} d_{\alpha} E_{k}\left(Y-d^{2}\right)\right. \\
& \left.\left.+E_{i} d_{\alpha} E_{j}\left(Y-d^{2}\right) E_{k} d_{\alpha}+E_{i}\left(Y-d^{2}\right) E_{j} d_{\alpha} E_{k} d_{\alpha}\right]+\frac{1}{6} \sum_{i, j, k, l} J_{i j k l} \delta_{\alpha \beta \gamma \delta} \operatorname{tr}_{D f}\left[E_{i} d_{\alpha} E_{j} d_{\beta} E_{k} d_{\gamma} E_{l} d_{\delta}\right]\right\}+\mathcal{O}\left(1 / M^{2}\right) \\
\equiv & -\frac{N_{c}}{32 \pi^{2}} \int d^{4} x \sum_{n=0}^{\infty} \operatorname{tr}_{D} b_{n}(x, x),
\end{aligned}
$$

where the index $n$ indicates the asymptotic behavior of $b_{n}(x, x)$ at large masses, namely $b_{n} \sim M^{-2(n-2)}$. The coefficients $b_{n}(x, x)$ depend on the external fields and quark masses, i.e., they contain information about both the effective meson vertices and corresponding coupling constants. If all masses are equal, the dependence on $m$ is factorized in form of the integral (18) and the fielddependent part takes a standard Seeley-DeWitt form $a_{n}(x, x)$.

\section{LEADING TERMS OF THE 1/M EXPANSION}

Consider the leading terms $b_{1}$ and $b_{2}$. The case $n=0$ is of no interest because $b_{0}$ contains no fields and can be omitted from the effective action. The coefficients with $n \geq 3$ tend to zero in the limit of infinite masses; therefore, they are small in comparison with $b_{1}$ and $b_{2}$ and, at the first stage, can be neglected.

\section{A. Coefficient $b_{1}(x, x)$}

Let us turn to the calculation of the coefficient $b_{1}$. It is given by a part of the expression (28), which is proportional to the proper-time integrals $J_{0}\left(m_{i}^{2}, m_{j}^{2}\right)$. With the use of Eq. (E1), it can be rewritten as

$$
\begin{aligned}
b_{1}(x, x)= & -\sum_{i} J_{0}\left(m_{i}^{2}\right) \operatorname{tr}_{f}\left[E_{i}\left(Y-d^{2}\right)+E_{i} d_{\alpha} E_{i} d_{\alpha}\right] \\
& -2 \sum_{i<j} J_{0}\left(m_{i}^{2}, m_{j}^{2}\right) \operatorname{tr}_{f}\left(E_{i} d_{\alpha} E_{j} d_{\alpha}\right) .
\end{aligned}
$$

Noting that

$$
d^{2}=\partial^{2}+2 i \Gamma \partial+i(\partial \Gamma)-\Gamma^{2},
$$

and taking into account that the action of the open derivatives in (29) on the implied unit on the right-hand side of Eq. (28) gives zero, we find

$$
\operatorname{tr}_{f}\left[E_{i}\left(Y-d^{2}\right)\right]=\operatorname{tr}_{f}\left[E_{i}\left(Y+\Gamma^{2}-i \partial \Gamma\right)\right] .
$$

Since the last term here is a total divergence which can be omitted in the effective action, we conclude

$\operatorname{tr}_{f}\left[E_{i}\left(Y-d^{2}\right)\right]=\operatorname{tr}_{f}\left[E_{i}\left(Y+\Gamma^{2}\right)\right]=Y_{i i}+\left(\Gamma^{2}\right)_{i i}$,

where in the last stage we used Eq. (12) and the fact that matrix $A$ may be written in a unique way as a finite linear combination of elements of $A$ in the bases $E_{i j}$, namely $A=\sum_{m, n} A_{m n} E_{m n}$. In the same manner, we find

$$
\operatorname{tr}_{f}\left(E_{i} d_{\alpha} E_{j} d_{\alpha}\right)=-\operatorname{tr}_{f}\left(E_{i} \Gamma_{\alpha} E_{j} \Gamma_{\alpha}\right)=-\Gamma_{i j}^{\alpha} \Gamma_{j i}^{\alpha} .
$$

This gives for Eq. (29)

$$
\begin{aligned}
b_{1}= & -\sum_{i} J_{0}\left(m_{i}^{2}\right)\left[Y_{i i}+\left(\Gamma^{2}\right)_{i i}-\Gamma_{i i}^{\alpha} \Gamma_{i i}^{\alpha}\right] \\
& +2 \sum_{i<j} J_{0}\left(m_{i}^{2}, m_{j}^{2}\right) \Gamma_{i j}^{\alpha} \Gamma_{j i}^{\alpha} .
\end{aligned}
$$

Let us use now the easily verifiable relation

$$
\left(\Gamma^{2}\right)_{i i}-\Gamma_{i i}^{\alpha} \Gamma_{i i}^{\alpha}=\sum_{j \neq i} \Gamma_{i j}^{\alpha} \Gamma_{j i}^{\alpha},
$$

to obtain finally

$$
b_{1}=-\sum_{i} J_{0}\left(m_{i}^{2}\right) Y_{i i}+\sum_{i<j} \Delta J_{0}\left(m_{i}^{2}, m_{j}^{2}\right) \Gamma_{i j}^{\alpha} \Gamma_{j i}^{\alpha},
$$

where $\Delta J_{0}\left(m_{i}^{2}, m_{j}^{2}\right)$ is given by Eq. (D14). The latter integrals can be collected in the symmetric $3 \times 3$ matrix $\Delta J_{0}$, which has zeros on the main diagonal. In particular, when $m_{i}=m_{j}=m$, all elements of $\Delta J_{0}$ vanish. In this specific case, the first term of (36) leads to the well-known expression of the Seeley-DeWitt coefficient $a_{1}(x, x)=-Y$.

For convenience of writing the result of our calculations, along with the usual matrix multiplication, we will use the nonstandard Hadamard product [41], which is the matrix of elementwise products

$$
(A \circ B)_{i j}=A_{i j} B_{i j} .
$$


The Hadamard product is commutative unlike regular matrix multiplication, but the distributive and associative properties are retained. It has previously been proven to be a useful tool when the mass matrices of the type (6) are involved [42]. In terms of the Hadamard product the result (36) can be written as

$$
b_{1}=\operatorname{tr}_{f}\left[J_{0} \circ(-Y)+\frac{1}{4}\left(\Delta J_{0} \circ \Gamma^{\alpha}\right) \Gamma^{\alpha}\right] \text {, }
$$

where $J_{0}$ is considered as a diagonal matrix with elements given by $\left(J_{0}\right)_{i j}=\delta_{i j} J_{0}\left(m_{i}^{2}\right)$. This matrix contains contributions of the Feynman one-loop diagram, known as a "tadpole."

\section{B. Coefficient $\boldsymbol{b}_{\mathbf{2}}(\boldsymbol{x}, \boldsymbol{x})$}

Consider the second coefficient $b_{2}(x, x)$. In accordance with the general structure of the expression, we distinguish three contributions differing in the degree of $Y$ :

$$
b_{2}=b_{2}^{(0)}+b_{2}^{(1)}+b_{2}^{(2)}
$$

which is explicitly indicated in the parentheses.

\section{Quadratic part in $Y: b_{2}^{(2)}$}

The part of $b_{2}$ proportional to $Y^{2}$ is calculated most simply:

$$
\begin{aligned}
b_{2}^{(2)} & =\frac{1}{2} \sum_{i, j} J_{i j} \operatorname{tr}_{f}\left(E_{i} Y E_{j} Y\right)=\frac{1}{2} \sum_{i, j} J_{i j} Y_{i j} Y_{j i} \\
& =\frac{1}{2} \operatorname{tr}_{f}[(J \circ Y) Y],
\end{aligned}
$$

where $J$ is a symmetric $3 \times 3$ matrix, whose elements $J_{i j}=$ $J_{1}\left(m_{i}, m_{j}\right)$ are logarithmically divergent parts (at $\Lambda \rightarrow \infty$ ) of Feynman self-energy diagrams with masses of virtual particles $m_{i}$ and $m_{j}$ [see Eq. (D6)].

\section{Linear part in $Y: b_{2}^{(1)}$}

Let us consider now the linear in $Y$ part of $b_{2}$, i.e., $b_{2}^{(1) \text { : }}$

$$
\begin{aligned}
b_{2}^{(1)}= & -\frac{1}{2} \sum_{i, j} J_{i j} \operatorname{tr}_{f}\left(E_{i} d^{2} E_{j} Y+E_{i} Y E_{j} d^{2}\right) \\
& +\frac{1}{3} \sum_{i, j, k} J_{i j k} \operatorname{tr}_{f}\left(E_{i} d_{\alpha} E_{j} d_{\alpha} E_{k} Y\right. \\
& \left.+E_{i} d_{\alpha} E_{j} Y E_{k} d_{\alpha}+E_{i} Y E_{j} d_{\alpha} E_{k} d_{\alpha}\right) .
\end{aligned}
$$

After evaluation of traces it gives

$$
b_{2}^{(1)}=-\frac{1}{2} \sum_{i, j} J_{i j} t_{i j}+\sum_{i, j, k} J_{i j k} t_{i j k},
$$

where

$$
t_{i j}=\left\{\left(d^{2}\right)_{i j}, Y_{j i}\right\}, \quad t_{i j k}=\left\langle d_{i j}^{\alpha} d_{j k}^{\alpha} Y_{k i}\right\rangle
$$

Next, let us use Eqs. (E1) and (E6) of Appendix E. We obtain

$$
\begin{aligned}
b_{2}^{(1)}= & \sum_{i} J_{i}\left(t_{i i i}-\frac{1}{2} t_{i i}\right)+\sum_{i<j} J_{i j}\left(3 t_{i i j}^{+}-t_{(i j)}\right) \\
& +3 \sum_{i<j} R_{i j} t_{i i j}^{-}+6 J_{123} t_{(123)} .
\end{aligned}
$$

To evaluate the first sum in this expression, we employ the explicit form of $t$ coefficients (43)

$$
\begin{aligned}
t_{i i i}-\frac{1}{2} t_{i i} & =\left\{\frac{1}{3} d_{i i}^{\alpha} d_{i i}^{\alpha}-\frac{1}{2}\left(d^{2}\right)_{i i}, Y_{i i}\right\}+\frac{1}{3} d_{i i}^{\alpha} Y_{i i} d_{i i}^{\alpha} \\
& =-\frac{1}{6}\left[d_{i i}^{\alpha},\left[d_{i i}^{\alpha}, Y_{i i}\right]\right]-\frac{1}{2} \sum_{j \neq i}\left\{d_{i j}^{\alpha} d_{j i}^{\alpha}, Y_{i i}\right\} .
\end{aligned}
$$

The first term is now computed as

$$
\left[d_{i i}^{\alpha},\left[d_{i i}^{\alpha}, Y_{i i}\right]\right]=\partial_{\alpha}^{2} Y_{i i}
$$

This is a four-divergence of a four-vector. Therefore, it can be omitted from the effective action, because it does not affect the dynamic characteristics of the effective theory. Thus, we find

$$
t_{i i i}-\frac{1}{2} t_{i i}=\sum_{j \neq i} \Gamma_{i j}^{\alpha} \Gamma_{j i}^{\alpha} Y_{i i}
$$

where we used the cyclic permutation of the elements to simplify the right-hand side of this expression, which is not forbidden due to the trace, $\operatorname{tr}_{D}$, in (28). In what follows, we will always use the cyclic permutation property when calculating the $t$ coefficients.

To evaluate the second sum in (44) we need to find the difference $3 t_{i i j}^{+}-t_{(i j)}$, where $i \neq j$. Here we have 


$$
\begin{aligned}
3 t_{i i j}^{+}-t_{(i j)} & =3\left(t_{(i i j)}+t_{(j j i)}\right)-\frac{1}{2}\left(t_{i j}+t_{j i}\right) \\
& =t_{i i j}+t_{i j i}+t_{j i i}-\frac{1}{2} t_{i j}+(i \leftrightarrow j) \\
& =\frac{1}{3}\left(\left\{d_{i i}^{\alpha} d_{i j}^{\alpha}+d_{i j}^{\alpha} d_{j j}^{\alpha}, Y_{j i}\right\}+d_{i j}^{\alpha} Y_{j i} d_{i i}^{\alpha}+d_{j j}^{\alpha} Y_{j i} d_{i j}^{\alpha}+\left\{d_{i j}^{\alpha} d_{j i}^{\alpha}, Y_{i i}\right\}+d_{j i}^{\alpha} Y_{i i} d_{i j}^{\alpha}\right)-\frac{1}{2}\left\{\left(d^{2}\right)_{i j}, Y_{j i}\right\}+(i \leftrightarrow j) \\
& =-\frac{1}{6}\left[\left\{d_{i i}^{\alpha} d_{i j}^{\alpha}+d_{i j}^{\alpha} d_{j j}^{\alpha}, Y_{j i}\right\}-2\left(d_{i j}^{\alpha} Y_{j i} d_{i i}^{\alpha}+d_{j j}^{\alpha} Y_{j i} d_{i j}^{\alpha}\right)\right]+\frac{1}{3}\left(\left\{d_{i j}^{\alpha} d_{j i}^{\alpha}, Y_{i i}\right\}+d_{j i}^{\alpha} Y_{i i} d_{i j}^{\alpha}\right)-\left.\frac{1}{2}\left\{d_{i k}^{\alpha} d_{k j}^{\alpha}, Y_{j i}\right\}\right|_{k \neq i, j}+(i \leftrightarrow j),
\end{aligned}
$$

where, in the last stage, we took into account that

$$
\left(d^{2}\right)_{i j}=\sum_{k} d_{i k}^{\alpha} d_{k j}^{\alpha}
$$

Noticing that expressions

$$
\left\{d_{i i}^{\alpha} d_{i j}^{\alpha}+d_{i j}^{\alpha} d_{j j}^{\alpha}, Y_{j i}\right\}-2\left(d_{i j}^{\alpha} Y_{j i} d_{i i}^{\alpha}+d_{j j}^{\alpha} Y_{j i} d_{i j}^{\alpha}\right), \quad\left\{d_{i j}^{\alpha} d_{j i}^{\alpha}, Y_{i i}\right\}-2 d_{j i}^{\alpha} Y_{i i} d_{i j}^{\alpha}
$$

vanish under the Dirac matrix trace in (28), we finally obtain

$$
3 t_{i i j}^{+}-t_{(i j)}=-\Gamma_{i j}^{\alpha} \Gamma_{j i}^{\alpha} Y_{i i}+\Gamma_{i k}^{\alpha} \Gamma_{k j}^{\alpha} Y_{j i}+(i \leftrightarrow j),
$$

where $i \neq j \neq k$.

Since the first term in (51) has the same form as (47), it follows that

$$
\sum_{i} J_{i}\left(t_{i i i}-\frac{1}{2} t_{i i}\right)-\sum_{i<j} J_{i j}\left[\Gamma_{i j}^{\alpha} \Gamma_{j i}^{\alpha} Y_{i i}+(i \leftrightarrow j)\right]=\sum_{\substack{i, j \\ i \neq j}}\left(J_{i}-J_{i j}\right) \Gamma_{i j}^{\alpha} \Gamma_{j i}^{\alpha} Y_{i i} .
$$

We need now to calculate the coefficient

$$
\begin{aligned}
t_{i i j}^{-} & =t_{(i i j)}-t_{(j j i)}=\frac{1}{3}\left[t_{i i j}+t_{i j i}+t_{j i i}-(i \leftrightarrow j)\right] \\
& =-\frac{1}{3}\left[\frac{i}{3}\left(\Gamma_{i j}^{\alpha} \stackrel{\leftrightarrow}{\partial_{\alpha}} Y_{j i}\right)+\left(\Gamma_{i i}^{\alpha} \Gamma_{i j}^{\alpha}-\Gamma_{i j}^{\alpha} \Gamma_{j j}^{\alpha}\right) Y_{j i}+\Gamma_{i j}^{\alpha} \Gamma_{j i}^{\alpha} Y_{i i}\right]-(i \leftrightarrow j),
\end{aligned}
$$

where the left-right derivative is defined in a standard way, $\Gamma^{\alpha} \stackrel{\leftrightarrow}{\partial^{\alpha}} Y=\Gamma^{\alpha} \partial^{\alpha} Y-\left(\partial^{\alpha} \Gamma^{\alpha}\right) Y$. Then the third sum in (44) is

$$
3 \sum_{i<j} R_{i j} t_{i i j}^{-}=-\sum_{i, j} R_{i j}\left[\frac{i}{3}\left(\Gamma_{i j}^{\alpha} \stackrel{\leftrightarrow}{\partial} Y_{j i}\right)+\left(\Gamma_{i i}^{\alpha} \Gamma_{i j}^{\alpha}-\Gamma_{i j}^{\alpha} \Gamma_{j j}^{\alpha}\right) Y_{j i}+\Gamma_{i j}^{\alpha} \Gamma_{j i}^{\alpha} Y_{i i}\right] .
$$

The coefficients $t_{(123)}$ are required for the evaluation of the last term in (44). They can be obtained with the aid of the easily verifiable relations,

$$
\left.t_{i j k}\right|_{i \neq j \neq k}=-\Gamma_{i j}^{\alpha} \Gamma_{j k}^{\alpha} Y_{k i}
$$

As a consequence, we have

$$
t_{(123)}=\frac{1}{6} \sum_{i \neq j \neq k} t_{i j k}=-\frac{1}{6} \sum_{i \neq j \neq k} \Gamma_{i j}^{\alpha} \Gamma_{j k}^{\alpha} Y_{k i}
$$


If we take into account the existence of similar term in the coefficient (51), we obtain

$$
6 J_{123} t_{(123)}+\sum_{\substack{i<j \\ i \neq j \neq k}} J_{i j}\left[\Gamma_{i k}^{\alpha} \Gamma_{k j}^{\alpha} Y_{j i}+(i \leftrightarrow j)\right]=\sum_{i \neq j \neq k}\left(J_{i j}-J_{i j k}\right) \Gamma_{i j}^{\alpha} \Gamma_{j k}^{\alpha} Y_{k i} .
$$

Thus, the final result for $b_{2}^{(1)}$ is

$$
\begin{aligned}
b_{2}^{(1)} & =\sum_{i \neq j \neq k}\left[\left(J_{i}-J_{i j}\right) \Gamma_{i j}^{\alpha} \Gamma_{j i}^{\alpha} Y_{i i}+\left(J_{i j}-J_{i j k}\right) \Gamma_{i j}^{\alpha} \Gamma_{j k}^{\alpha} Y_{k i}\right]-\sum_{i, j} R_{i j}\left[\frac{i}{3}\left(\Gamma_{i j}^{\alpha} \stackrel{\leftrightarrow}{\partial^{\alpha}} Y_{j i}\right)+\left(L_{-}^{\alpha \alpha}\right)_{i j} Y_{j i}+\Gamma_{i j}^{\alpha} \Gamma_{j i}^{\alpha} Y_{i i}\right] \\
& =\operatorname{tr}_{f}\left[N Y-\frac{i}{3}\left(R \circ \Gamma^{\alpha}\right) \stackrel{\leftrightarrow}{\partial^{\alpha}} Y\right]
\end{aligned}
$$

where we introduce the notation

$$
\left(L_{ \pm}^{\alpha \beta}\right)_{i j}=\left(\Gamma_{i i}^{\alpha} \pm \Gamma_{j j}^{\alpha}\right) \Gamma_{i j}^{\beta} .
$$

Note that elements of the matrix $\Gamma_{i j}^{\alpha}$ commute with each other. In the last line of Eq. (58), $R$ and $N$ are the $3 \times 3$ matrices with elements

$$
\begin{aligned}
& R_{i j}=\frac{1}{2}\left(J_{i i j}-J_{j j i}\right), \\
& N_{i i}=\sum_{j \neq i}\left(J_{i}-J_{i j}-R_{i j}\right) \Gamma_{i j}^{\alpha} \Gamma_{j i}^{\alpha}, \\
& N_{i j}=\left[\left(J_{i j}-J_{i j k}\right) \Gamma_{i k}^{\alpha} \Gamma_{k j}^{\alpha}-R_{i j}\left(L_{-}^{\alpha \alpha}\right)_{i j}\right]_{\mid i \neq j \neq k} .
\end{aligned}
$$

In the limit of equal masses, the coefficient $b_{2}^{(1)}$ vanishes, that is, it contains only contributions associated with an explicit violation of chiral symmetry. Obviously, without this term, the description of the manifest flavor symmetry breaking would be incomplete.

\section{Independent of $Y$ part: $b_{2}^{(0)}$}

The part of $b_{2}$ which does not depend on $Y$ is

$b_{2}^{(0)}=\frac{1}{2} \sum_{i, j} J_{i j}\left(d^{2}\right)_{i j}\left(d^{2}\right)_{j i}-\sum_{i, j, k} J_{i j k} \hat{t}_{i j k}+\frac{1}{6} \sum_{i, j, k, l} J_{i j k l} t_{i j k l}$.

Here the field-dependent $t$ coefficients are given by the following formulas:

$$
\hat{t}_{i j k}=\left\langle d_{i j}^{\alpha} d_{j k}^{\alpha}\left(d^{2}\right)_{k i}\right\rangle, \quad t_{i j k l}=\delta_{\alpha \beta \gamma \delta}\left\langle d_{i j}^{\alpha} d_{j k}^{\beta} d_{k l}^{\gamma} d_{l i}^{\delta}\right\rangle .
$$

Before calculating (61) directly, we shall first handle the case of equal masses $m_{u}=m_{d}=m_{s}=m$, which can easily be obtained from (61) after factorizing the common factor $J_{1}\left(m^{2}\right)=J_{i i}=J_{i i i}=J_{i i i i}$ :

$$
\begin{aligned}
\frac{\left.b_{2}^{(0)}\right|_{m}}{J_{1}\left(m^{2}\right)}= & \operatorname{tr}_{f}\left[\frac{1}{2} d^{2} d^{2}-\frac{1}{3}\left(d^{\alpha} d^{\alpha} d^{2}+d^{\alpha} d^{2} d^{\alpha}+d^{2} d^{\alpha} d^{\alpha}\right)\right. \\
& \left.+\frac{1}{6}\left(d^{\alpha} d^{\alpha} d^{\beta} d^{\beta}+d^{\alpha} d^{\beta} d^{\alpha} d^{\beta}+d^{\alpha} d^{\beta} d^{\beta} d^{\alpha}\right)\right] \\
= & \frac{1}{6} \operatorname{tr}_{f}\left(d^{\alpha} d^{\beta} d^{\alpha} d^{\beta}-d^{\alpha} d^{\beta} d^{\beta} d^{\alpha}\right)=\frac{1}{12} \operatorname{tr}_{f}\left[d^{\alpha}, d^{\beta}\right]^{2} \\
= & -\frac{1}{12} \operatorname{tr}_{f}\left(\Gamma^{\alpha \beta} \Gamma^{\alpha \beta}\right),
\end{aligned}
$$

where we used Eq. (49), and

$$
\Gamma^{\alpha \beta}=\partial^{\alpha} \Gamma^{\beta}-\partial^{\beta} \Gamma^{\alpha}+i\left[\Gamma^{\alpha}, \Gamma^{\beta}\right] .
$$

To obtain the exact result for (61) it is convenient to consider separately the cases when the sum runs only over the same index values, and then over two and three different values of the flavors:

$$
b_{2}^{(0)}=\beta_{2}^{(1)}+\beta_{2}^{(2)}+\beta_{2}^{(3)} .
$$

The part of $b_{2}^{(0)}$ that corresponds to a contribution with coincident indices is

$$
\beta_{2}^{(1)}=\sum_{i} J_{i}\left[\frac{1}{2}\left(d^{2}\right)_{i i}\left(d^{2}\right)_{i i}-\hat{t}_{i i i}+\frac{1}{6} t_{i i i i}\right] .
$$

Introducing the notation

$$
Q_{i i}=d_{i i}^{\alpha} d_{i i}^{\alpha}-\left(d^{2}\right)_{i i}=\left(\Gamma^{2}\right)_{i i}-\Gamma_{i i}^{\alpha} \Gamma_{i i}^{\alpha},
$$

we can rewrite (66) as

$$
\beta_{2}^{(1)}=\sum_{i} J_{i}\left\{\frac{1}{2} Q_{i i}^{2}-\frac{1}{6}\left[d_{i i}^{\alpha},\left[d_{i i}^{\alpha}, Q_{i i}\right]\right]+\frac{1}{12}\left[d_{i i}^{\alpha}, d_{i i}^{\beta}\right]^{2}\right\} .
$$

Now we benefit from the following easily established relations: 


$$
\begin{gathered}
{\left[d_{i i}^{\alpha},\left[d_{i i}^{\alpha}, Q_{i i}\right]\right]=\partial^{2} Q_{i i},} \\
{\left[d_{i i}^{\alpha}, d_{i i}^{\beta}\right]=i\left(\partial^{\alpha} \Gamma_{i i}^{\beta}-\partial^{\beta} \Gamma_{i i}^{\alpha}\right) \equiv i F_{i i}^{\alpha \beta} .}
\end{gathered}
$$

That gives finally

$$
\beta_{2}^{(1)}=\sum_{i} J_{i}\left\{\frac{1}{2} Q_{i i}^{2}-\frac{1}{12}\left(F_{i i}^{\alpha \beta}\right)^{2}\right\} .
$$

To obtain this expression, we as before discarded a full divergence of a four-vector $\partial^{2} Q_{i i}$.

Now consider the terms of the sum (61) with two unequal indices. These comprise the remaining terms of the first sum and the corresponding contributions from the second and third sums:

$$
\begin{aligned}
\beta_{2}^{(2)}= & \frac{1}{2} \sum_{i<j} J_{i j}\left\{\left(d^{2}\right)_{i j},\left(d^{2}\right)_{j i}\right\}-3 \sum_{i<j}\left(J_{i j} \hat{t}_{i i j}^{+}+R_{i j} \hat{t}_{i i j}^{-}\right) \\
& +\sum_{i<j}\left[\frac{2}{3}\left(J_{i j}+\frac{1}{2} S_{i j}\right) t_{i i i j}^{+}+R_{i j} t_{i i i j}^{-}\right] \\
& +\sum_{i<j}\left(J_{i j}-S_{i j}\right) t_{(i i j j)}
\end{aligned}
$$

where $S_{i j}$ is given by Eq. (D26).

To evaluate the first sum, we note that

$$
\begin{aligned}
\left(d^{2}\right)_{i j}\left(d^{2}\right)_{j i}= & \left(\partial \Gamma_{i j}\right)\left(\partial \Gamma_{j i}\right)+\left(\Gamma^{2}\right)_{i j}\left(\Gamma^{2}\right)_{j i} \\
& +i\left(\partial \Gamma_{i j}\right)\left(\Gamma^{2}\right)_{j i}-i\left(\Gamma^{2}\right)_{i j}\left(\partial \Gamma_{j i}\right) .
\end{aligned}
$$

Here we have omitted a full divergence of a four-vector, and took into account that the action of the derivative that reached the right end of the expression gives zero. In the anticommutator $\left\{\left(d^{2}\right)_{i j},\left(d^{2}\right)_{j i}\right\}$, only the symmetric part of Eq. (72) contributes to the sum. Therefore, the second line of (72) has no influence on the result, and we obtain

$$
\begin{aligned}
& \frac{1}{2} \sum_{i<j} J_{i j}\left\{\left(d^{2}\right)_{i j},\left(d^{2}\right)_{j i}\right\} \\
& \quad=\sum_{i<j} J_{i j}\left[\left(\partial \Gamma_{i j}\right)\left(\partial \Gamma_{j i}\right)+\left(\Gamma^{2}\right)_{i j}\left(\Gamma^{2}\right)_{j i}\right] .
\end{aligned}
$$

Recall again that elements of the matrix $\Gamma$ commute with each other.

Combining

$$
\frac{2}{3} t_{i i i j}^{+}+t_{(i i j j)}=\frac{2}{3}\left(t_{i i i j}+t_{j j j i}+t_{i i j j}\right)+\frac{1}{3} t_{i j i j},
$$

where the properties of $t$ coefficients (F12)-(F14) have been used, we learn that this sum does not contain the terms with one derivative. Indeed,

$$
\begin{aligned}
t_{i i i j}+t_{j j j i}+t_{i i j j}= & \left(\partial \Gamma_{i j}\right)\left(\partial \Gamma_{j i}\right)+\frac{1}{2}\left(\partial^{\alpha} \Gamma_{i j}^{\beta}\right)\left(\partial^{\alpha} \Gamma_{j i}^{\beta}\right) \\
& +\delta_{\alpha \beta \gamma \sigma}\left(\Gamma_{i i}^{\alpha} \Gamma_{i i}^{\beta} \Gamma_{i j}^{\gamma} \Gamma_{j i}^{\sigma}+\Gamma_{j j}^{\alpha} \Gamma_{j j}^{\beta} \Gamma_{j i}^{\gamma} \Gamma_{i j}^{\sigma}\right. \\
& \left.+\Gamma_{i i}^{\alpha} \Gamma_{i j}^{\beta} \Gamma_{j j}^{\gamma} \Gamma_{j i}^{\sigma}\right) .
\end{aligned}
$$

We also know from (F10) that

$$
t_{i j i j}=\delta_{\alpha \beta \gamma \sigma} \Gamma_{i j}^{\alpha} \Gamma_{j i}^{\beta} \Gamma_{i j}^{\gamma} \Gamma_{j i}^{\sigma} \text {. }
$$

Thus, only the coefficient $\hat{t}_{i i j}^{+}$contains terms with one derivative. The expression for this coefficient is given in Appendix F [see Eq. (F7)].

Before we turn to the full expression for the coefficient $\beta_{2}^{(2)}$ let us first collect terms with two derivatives in $\beta_{2}^{(2)}$ :

$$
\begin{aligned}
\left.\beta_{2}^{(2)}\right|_{\partial^{2}} & =\frac{1}{3} \sum_{i<j} J_{i j}\left[\left(\partial \Gamma_{i j}\right)\left(\partial \Gamma_{j i}\right)-\left(\partial^{\alpha} \Gamma_{i j}^{\beta}\right)\left(\partial^{\alpha} \Gamma_{j i}^{\beta}\right)\right] \\
& =-\frac{1}{6} \sum_{i<j} J_{i j} F_{i j}^{\alpha \beta} F_{j i}^{\alpha \beta} .
\end{aligned}
$$

Combined together with similar terms in $\beta_{2}^{(1)}$ they give the kinetic term of spin-1 fields:

$$
\begin{aligned}
\left.\left(\beta_{2}^{(1)}+\beta_{2}^{(2)}\right)\right|_{\partial^{2}}= & -\frac{1}{12} \operatorname{tr}_{f}\left[F^{\alpha \beta}\left(J \circ F^{\alpha \beta}\right)\right] \\
= & -\frac{1}{12} \operatorname{tr}_{f}\left[\Gamma^{\alpha \beta}\left(J \circ \Gamma^{\alpha \beta}\right)\right]+\frac{i}{3} \operatorname{tr}_{f}\left[F^{\alpha \beta}\left(J \circ \Gamma^{\alpha} \Gamma^{\beta}\right)\right] \\
& -\frac{1}{6} \operatorname{tr}_{f}\left\{\left[\Gamma^{\alpha}, \Gamma^{\beta}\right]\left(J \circ \Gamma^{\alpha} \Gamma^{\beta}\right)\right\} .
\end{aligned}
$$

In the case of equal masses, the first term coincides with the standard result (63). It means that the last two terms of (77), after combining them with other contributions to $b_{2}^{(0)}$, should vanish when $m_{u}=m_{d}=m_{s}=m$.

To see this we need to consider the contribution $\beta_{2}^{(3)}$. Referring to Eqs. (61) and (E7), we find

$$
\beta_{2}^{(3)}=2 \sum_{\substack{i \neq j \neq k \\ j<k}} J_{i i j k} t_{(i i j k)}-6 J_{123} \hat{t}_{(123)} .
$$

Using the explicit expression for the coefficients $t_{(i i j k)}$ (F19) and Eq. (D22), one can represent the first sum as 


$$
\begin{aligned}
2 \sum_{\substack{i \neq j \neq k \\
j<k}} J_{i i j k} t_{(i i j k)} & =\sum_{i \neq j \neq k} J_{i i j k} t_{(i i j k)} \\
& =\frac{1}{3} \delta_{\alpha \beta \gamma \sigma} \sum_{i \neq j \neq k}\left[\frac{i}{2}\left(J_{j j i k}-J_{i i j k}\right)\left(\partial^{\alpha} \Gamma_{i j}^{\beta}\right) \Gamma_{j k}^{\gamma} \Gamma_{k i}^{\sigma}+J_{i i j k}\left(\Gamma_{i i}^{\alpha} \Gamma_{i j}^{\beta} \Gamma_{j k}^{\gamma} \Gamma_{k i}^{\sigma}+\Gamma_{i i}^{\alpha} \Gamma_{i k}^{\beta} \Gamma_{k j}^{\gamma} \Gamma_{j i}^{\sigma}+\Gamma_{i j}^{\alpha} \Gamma_{j i}^{\beta} \Gamma_{i k}^{\gamma} \Gamma_{k i}^{\sigma}\right)\right] .
\end{aligned}
$$

If we introduce the antisymmetric matrix $K$ with elements

$$
K_{i j}=\left(J_{j j i k}-J_{i i j k}\right)_{\mid i \neq j \neq k},
$$

then the first part of the expression can be presented in the index-free form

$$
\frac{i}{6} \delta_{\alpha \beta \gamma \sigma} \operatorname{tr}_{f}\left[\left(K \circ \partial^{\alpha} \Gamma^{\beta}\right) E^{\gamma \sigma}\right]
$$

where the off-diagonal elements of matrix $E^{\gamma \sigma}$ are

$$
E_{i j}^{\gamma \sigma}=\Gamma_{i k}^{\gamma} \Gamma_{k j}^{\sigma}, \quad(i \neq j \neq k)
$$

Due to Eq. (79), the diagonal elements of the matrix $E^{\gamma \sigma}$ do not contribute to $(80)$.

It is also clear from (79) that terms with one derivative (80) vanish in the limit of equal masses. Therefore, they cannot cancel the unwanted second term in (77).

Since the coefficient $\hat{t}_{(123)}$ is known from Eq. (F9), we can give a complete expression for $\beta_{2}^{(3)}$ :

$$
\begin{aligned}
\beta_{2}^{(3)}= & \frac{1}{3} \delta_{\alpha \beta \gamma \sigma} \sum_{i \neq j \neq k}\left[\frac{i}{2}\left(J_{j j i k}-J_{i i j k}\right)\left(\partial^{\alpha} \Gamma_{i j}^{\beta}\right) \Gamma_{j k}^{\gamma} \Gamma_{k i}^{\sigma}\right. \\
& \left.+J_{i i j k}\left(\Gamma_{i i}^{\alpha} \Gamma_{i j}^{\beta} \Gamma_{j k}^{\gamma} \Gamma_{k i}^{\sigma}+\Gamma_{i i}^{\alpha} \Gamma_{i k}^{\beta} \Gamma_{k j}^{\gamma} \Gamma_{j i}^{\sigma}+\Gamma_{i j}^{\alpha} \Gamma_{j i}^{\beta} \Gamma_{i k}^{\gamma} \Gamma_{k i}^{\sigma}\right)\right] \\
& -J_{123} \sum_{i \neq j \neq k}\left[\frac{i}{3} \Gamma_{i j}^{\alpha} \Gamma_{j k}^{\beta} F_{k i}^{\alpha \beta}+\Gamma_{i j}^{\alpha} \Gamma_{j k}^{\alpha}\left(\Gamma^{2}\right)_{k i}\right] .
\end{aligned}
$$

Now it is necessary to make sure that the terms linear in $F^{\alpha \beta}$ vanish in the case of equal fermion masses. To this end let us consider the corresponding contributions to $b_{2}^{(0)}$ from $\hat{t}_{(i i j)}^{+}$in (71), from the second term of (77), and from $\hat{t}_{(123)}$ of Eq. (78). Combined together they give

$$
\begin{aligned}
& \frac{i}{3} \sum_{i \neq j \neq k}\left[\left(J_{i}-J_{i j}\right) \Gamma_{i j}^{\alpha} \Gamma_{j i}^{\beta} F_{i i}^{\alpha \beta}+\left(J_{i j}-J_{123}\right) \Gamma_{i j}^{\alpha} \Gamma_{j k}^{\beta} F_{k i}^{\alpha \beta}\right] \\
& \quad=\frac{i}{3} \operatorname{tr}_{f}\left(H^{\alpha \beta} F^{\alpha \beta}\right)
\end{aligned}
$$

where the flavor matrix $H^{\alpha \beta}$ has elements

$$
\begin{aligned}
H_{i i}^{\alpha \beta} & =\sum_{j \neq i}\left(J_{i}-J_{i j}\right) \Gamma_{i j}^{\alpha} \Gamma_{j i}^{\beta}, \\
\left.\left(H_{i j}^{\alpha \beta}\right)\right|_{i \neq j} & =\left(J_{i j}-J_{123}\right) E_{i j}^{\alpha \beta} .
\end{aligned}
$$

One can see that all elements of this matrix vanish in the limit of equal masses.

Our next step is to demonstrate the fulfilment of the limiting condition for terms of the fourth order in $\Gamma$. As we already know, these terms should also vanish for equalmass case.

In the first step, we transform the following expression:

$$
\begin{aligned}
& \frac{1}{2} \sum_{i} J_{i} Q_{i i}^{2}-J_{123} \sum_{i \neq j \neq k} \Gamma_{i j}^{\alpha} \Gamma_{j k}^{\alpha}\left(\Gamma^{2}\right)_{k i}+\sum_{i<j} J_{i j}\left(\Gamma^{2}\right)_{i j}\left(\Gamma^{2}\right)_{j i} \\
& -\sum_{i<j} J_{i j}\left[\Gamma_{j i}^{\alpha}\left(\Gamma_{i i}^{\alpha}+\Gamma_{j j}^{\alpha}\right)\left(\Gamma^{2}\right)_{i j}+\Gamma_{i j}^{\alpha} \Gamma_{j i}^{\alpha}\left(\Gamma^{2}\right)_{i i}+(i \leftrightarrow j)\right] .
\end{aligned}
$$

Here the first sum is taken from $\beta_{2}^{(1)}$ [see Eq. (70)], the second one from $\beta_{2}^{(3)}$ [the last term in Eq. (82)], and the third and fourth sums are contributions from $\beta_{2}^{(2)}$ [from the first and $\hat{t}_{i i j}^{+}$terms of (71), correspondingly].

This expression can be made into a somewhat more simple form:

$$
\frac{1}{2} \operatorname{tr}_{f}\left[\left(H^{\alpha \alpha}-\hat{H}^{\alpha \alpha}\right) \Gamma^{2}\right]+\frac{1}{2} \sum_{i} J_{i}\left(\Gamma_{i i} \Gamma_{i i}\right)^{2}
$$

where the matrix $H^{\alpha \alpha}$ is a particular case of (84), and the elements of the matrix $\hat{H}^{\alpha \alpha}$ are

$$
\begin{aligned}
\hat{H}_{i i}^{\alpha \alpha} & =\sum_{j} J_{i j} \Gamma_{i j}^{\alpha} \Gamma_{j i}^{\alpha}, \\
\left.\hat{H}_{i j}^{\alpha \alpha}\right|_{i \neq j} & =J_{i j} \Gamma_{i j}^{\alpha}\left(\Gamma_{i i}^{\alpha}+\Gamma_{j j}^{\alpha}\right)+J_{123} E_{i j}^{\alpha \alpha} .
\end{aligned}
$$

Under the flavor trace in (85), we explicitly singled out the contribution $H^{\alpha \alpha}$ that vanishes in the case of equal masses. The last term in (85) is completely canceled by the corresponding contribution from $\hat{H}^{\alpha \alpha}$. Indeed, it is straightforward to see 


$$
\begin{aligned}
- & \frac{1}{2} \operatorname{tr}_{f}\left(\hat{H}^{\alpha \alpha} \Gamma^{2}\right)+\frac{1}{2} \sum_{i} J_{i}\left(\Gamma_{i i} \Gamma_{i i}\right)^{2} \\
= & -\frac{1}{2} \sum_{i \neq j} \Gamma_{i j}^{\alpha} \Gamma_{j i}^{\alpha}\left[J_{i}\left(\Gamma_{i i} \Gamma_{i i}\right)+J_{i j}\left(\Gamma^{2}\right)_{i i}\right] \\
& -\frac{1}{2} \sum_{i \neq j} \hat{H}_{i j}^{\alpha \alpha}\left(\Gamma^{2}\right)_{j i} .
\end{aligned}
$$

$$
-\frac{1}{12} \sum_{i} J_{i}\left[\Gamma^{\alpha}, \Gamma^{\beta}\right]_{i i}^{2}-\frac{1}{6} \sum_{i<j} J_{i j}\left[\Gamma^{\alpha}, \Gamma^{\beta}\right]_{i j}\left[\Gamma^{\alpha}, \Gamma^{\beta}\right]_{j i},
$$

and the $\Gamma^{4}$ part of $\beta_{2}^{(3)}$ from Eq. (82)

At the second stage, we collect three contributions: the part of $\beta_{2}^{(2)}$ associated with the coefficient (74),

$$
\frac{1}{6} \delta_{\alpha \beta \gamma \sigma} \sum_{i \neq j} J_{i j} \Gamma_{i j}^{\alpha} \Gamma_{j i}^{\beta}\left(\Gamma_{i j}^{\gamma} \Gamma_{j i}^{\sigma}+2 \Gamma_{i i}^{\gamma} \Gamma_{j j}^{\sigma}+4 \Gamma_{i i}^{\gamma} \Gamma_{i i}^{\sigma}\right),
$$

the last term in Eq. (77),

$\frac{1}{3} \delta_{\alpha \beta \gamma \sigma} \sum_{i \neq j \neq k} J_{i i j k}\left(\Gamma_{i i}^{\alpha} \Gamma_{i j}^{\beta} \Gamma_{j k}^{\gamma} \Gamma_{k i}^{\sigma}+\Gamma_{i i}^{\alpha} \Gamma_{i k}^{\beta} \Gamma_{k j}^{\gamma} \Gamma_{j i}^{\sigma}+\Gamma_{i j}^{\alpha} \Gamma_{j i}^{\beta} \Gamma_{i k}^{\gamma} \Gamma_{k i}^{\sigma}\right)$.

Together with the terms just considered in (87), they should lead to a result that vanishes in the limit of equal masses.

Indeed, it is evident from the direct calculation of these terms that the summation gives 33 independent combinations of $\Gamma$ 's, which can be collected in the following sums:

$$
\begin{aligned}
\Omega_{1}^{(0)}= & (85)+(88)+(89)+(90) \\
= & \sum_{i<j}\left\{\left[\frac{2}{3}\left(J_{i}+J_{j}\right)-\frac{4}{3} J_{i j}\right]\left(\Gamma_{i j} \Gamma_{j i}\right)^{2}+\frac{1}{6}\left(2 J_{i j}-J_{i}-J_{j}\right)\left(\Gamma_{i j} \Gamma_{i j}\right)\left(\Gamma_{j i} \Gamma_{j i}\right)\right\} \\
& +\sum_{\substack{i \neq j \neq k \\
j<k}}\left\{\left[\frac{1}{3}\left(J_{j k}+2 J_{i i j k}\right)+J_{i}-J_{i j}-J_{i k}\right]\left(\Gamma_{i j} \Gamma_{j i}\right)\left(\Gamma_{i k} \Gamma_{k i}\right)+\left[\frac{1}{3}\left(J_{i}+2 J_{i i j k}\right)+J_{j k}-2 J_{123}\right]\left(\Gamma_{j i} \Gamma_{i k}\right)\left(\Gamma_{k i} \Gamma_{i j}\right)\right. \\
& \left.+\frac{1}{3}\left(2 J_{i i j k}-J_{i j}-J_{i k}\right)\left[\left(\Gamma_{i i} \Gamma_{j k}\right)\left(\Gamma_{i j} \Gamma_{k i}\right)+\left(\Gamma_{i k} \Gamma_{j i}\right)\left(\Gamma_{k j} \Gamma_{i i}\right)\right]+\frac{1}{3}\left(2 J_{i i j k}-J_{i}-J_{j k}\right)\left(\Gamma_{i j} \Gamma_{i k}\right)\left(\Gamma_{k i} \Gamma_{j i}\right)\right\} \\
& +\sum_{i \neq j \neq k}\left[\frac{1}{3}\left(J_{i k}+2 J_{i i j k}\right)-J_{123}\right]\left[\left(\Gamma_{i i} \Gamma_{i j}\right)\left(\Gamma_{j k} \Gamma_{k i}\right)+\left(\Gamma_{i k} \Gamma_{k j}\right)\left(\Gamma_{j i} \Gamma_{i i}\right)\right] .
\end{aligned}
$$

Here, the expression in the parentheses $\left(\Gamma_{i j} \Gamma_{j i}\right)$ is understood to be summed over alpha $\Gamma_{i j}^{\alpha} \Gamma_{j i}^{\alpha}$. In the case of equal quark masses (91) is zero, since all $J$-dependent factors vanish.

It remains to calculate the sums containing the coefficients $R_{i j}$ and $S_{i j}$ in (71). For that we need to know the coefficients $3 \hat{t}_{i i j}^{-}-t_{i i i j}^{-}$and $t_{i i i j}^{+}-3 t_{(i i j j)}$.

From Eqs. (F8) and (F16) one finds

$$
3 \hat{t}_{i i j}^{-}-t_{i i i j}^{-}=\frac{i}{12}\left[8\left(\partial \Gamma_{j i}\right)\left(\Gamma^{2}\right)_{i j}+F_{i i}^{\alpha \beta} \Gamma_{i j}^{\alpha} \Gamma_{j i}^{\beta}+\left(F_{i j}^{\alpha \beta}+9 \partial^{\alpha} \Gamma_{i j}^{\beta}\right) L_{+j i}^{\beta \alpha}\right]+L_{-i j}^{\alpha \alpha}\left(\Gamma_{j k} \Gamma_{k i}\right)+\left(\Gamma_{i j} \Gamma_{j i}\right)\left(\Gamma_{i k} \Gamma_{k i}\right)-(i \leftrightarrow j),
$$

where it is assumed that $k \neq i \neq j$. Then, due to the antisymmetry of $R_{i j}$, we find

$$
\sum_{i<j} R_{j i}\left(3 \hat{t}_{i i j}^{-}-t_{i i i j}^{-}\right)=\sum_{i \neq j} R_{i j}\left\{\frac{i}{12}\left[8\left(\partial \Gamma_{i j}\right)\left(\Gamma^{2}\right)_{j i}+\Gamma_{j i}^{\alpha} \Gamma_{i j}^{\beta} F_{j j}^{\alpha \beta}+\left(F_{j i}^{\alpha \beta}+9 \partial^{\alpha} \Gamma_{j i}^{\beta}\right) L_{+i j}^{\beta \alpha}\right]+L_{-j i}^{\alpha \alpha}\left(\Gamma_{i k} \Gamma_{k j}\right)+\left(\Gamma_{j i} \Gamma_{i j}\right)\left(\Gamma_{j k} \Gamma_{k j}\right)\right\} .
$$

Now, from Eqs. (F16) and (F18) one obtains

$$
\begin{aligned}
t_{i i i j}^{+}-3 t_{(i i j j)}= & \frac{1}{4} F_{i j}^{\alpha \beta} F_{j i}^{\alpha \beta}+\frac{3}{2}\left(\partial \Gamma_{i j}\right)\left(\partial \Gamma_{j i}\right)-\frac{3 i}{4}\left(\Gamma_{i i}^{\alpha}-\Gamma_{j j}^{\alpha}\right)\left[\left(\Gamma_{j i}^{\alpha} \stackrel{\leftrightarrow}{\beta}^{\beta} \Gamma_{i j}^{\beta}\right)+\left(\Gamma_{j i}^{\beta} \stackrel{\leftrightarrow}{\partial^{\alpha}} \Gamma_{i j}^{\beta}\right)+\left(\Gamma_{j i}^{\beta} \stackrel{\leftrightarrow}{\partial^{\beta}} \Gamma_{i j}^{\alpha}\right)\right] \\
& +\delta_{\alpha \beta \gamma \sigma}\left(\Gamma_{i i}^{\alpha} \Gamma_{i i}^{\beta} \Gamma_{i j}^{\gamma} \Gamma_{j i}^{\sigma}+\Gamma_{j j}^{\alpha} \Gamma_{j j}^{\beta} \Gamma_{j i}^{\gamma} \Gamma_{i j}^{\sigma}-\Gamma_{i j}^{\alpha} \Gamma_{j i}^{\beta} \Gamma_{i j}^{\gamma} \Gamma_{j i}^{\sigma}-2 \Gamma_{i i}^{\alpha} \Gamma_{i j}^{\beta} \Gamma_{j j}^{\gamma} \Gamma_{j i}^{\sigma}\right) .
\end{aligned}
$$

By virtue of the symmetry $S_{i j}=S_{j i}$, the required sum is 


$$
\begin{aligned}
\sum_{i<j} S_{i j}\left(\frac{1}{3} t_{i i i j}^{+}-t_{(i i j j)}\right)= & \frac{1}{3} \sum_{i \neq j} S_{i j}\left\{\frac{1}{8} F_{i j}^{\alpha \beta} F_{j i}^{\alpha \beta}+\frac{3}{4}\left(\partial \Gamma_{i j}\right)\left(\partial \Gamma_{j i}\right)-\frac{3 i}{8}\left(\Gamma_{i i}^{\alpha}-\Gamma_{j j}^{\alpha}\right)\left[\left(\Gamma_{j i}^{\alpha} \stackrel{\leftrightarrow}{\partial^{\beta}} \Gamma_{i j}^{\beta}\right)+\left(\Gamma_{j i}^{\beta} \stackrel{\leftrightarrow}{\partial^{\alpha}} \Gamma_{i j}^{\beta}\right)+\left(\Gamma_{j i}^{\beta} \stackrel{\leftrightarrow}{\partial^{\beta}} \Gamma_{i j}^{\alpha}\right)\right]\right. \\
& \left.+\delta_{\alpha \beta \gamma \sigma}\left(\Gamma_{i i}^{\alpha} \Gamma_{i i}^{\beta} \Gamma_{i j}^{\gamma} \Gamma_{j i}^{\sigma}-\Gamma_{i i}^{\alpha} \Gamma_{i j}^{\beta} \Gamma_{j j}^{\gamma} \Gamma_{j i}^{\sigma}-\frac{1}{2} \Gamma_{i j}^{\alpha} \Gamma_{j i}^{\beta} \Gamma_{i j}^{\gamma} \Gamma_{j i}^{\sigma}\right)\right\} .
\end{aligned}
$$

Now we have all the base ingredients that remain to be assembled into a single whole.

\section{Full result for $b_{2}(x, x)$}

Here we summarize the above results, collecting them in a compact form convenient for further applications. Namely, the result obtained for $b_{2}(x, x)$ can be represented as

$$
b_{2}=\frac{1}{2} \operatorname{tr}_{f}\left[Y(J \circ Y)-\frac{1}{6} \Gamma^{\alpha \beta}\left(J \circ \Gamma^{\alpha \beta}\right)\right]+\Delta b_{2} .
$$

In the limit of equal masses, the first two terms in (96) yield the well-known result [14], and the third one vanishes, that is, it contains only contributions associated with an explicit violation of chiral symmetry. Together with the second term of Eq. (36), this is the main result of our work.

To write the full expression for $\Delta b_{2}$, we distinguish four different contributions:

$$
\Delta b_{2}=b_{2}^{(1)}+\sum_{n=0}^{2} \Omega^{(n)} .
$$

Here $b_{2}^{(1)}$ is the sum of all terms linear in $Y$, and $\Omega^{(n)}$ is the sum of terms with $n$ derivatives which consists of only spin-1 fields, entering into $\Gamma^{\alpha}$.

For $b_{2}^{(1)}$ we have [see Eq. (58)]

$$
b_{2}^{(1)}=\operatorname{tr}_{f}\left[N Y-\frac{i}{3}\left(R \circ \Gamma^{\alpha}\right) \stackrel{\leftrightarrow}{\partial^{\alpha}} Y\right] .
$$

The terms with two derivatives are

$$
\Omega^{(2)}=\frac{1}{24} \operatorname{tr}_{f}\left[F^{\alpha \beta}\left(S \circ F^{\alpha \beta}\right)+6(\partial \Gamma)(S \circ \partial \Gamma)\right] .
$$

The first term makes an additional contribution to the kinetic part of the effective Lagrangian of spin-1 fields described by the second term in (96). Recall that the symmetric matrix $S$ is defined by $S_{i j}=J_{i j}-J_{i i j j}$ (D26). The shorthand $\partial \Gamma \equiv \partial^{\alpha} \Gamma^{\alpha}$ implies summation over omitted indices $\alpha$. In applications, one can omit the second term in (99) to ensure that the energy of the massive spin-1 field is positive definite.
The terms with one derivative can be collected in the expression

$$
\begin{aligned}
\Omega^{(1)}= & \frac{i}{3} \operatorname{tr}_{f}\left[H^{\alpha \beta} F^{\alpha \beta}+\frac{1}{2} \delta_{\alpha \beta \gamma \sigma}\left(K \circ \partial^{\alpha} \Gamma^{\beta}\right) E^{\gamma \sigma}\right] \\
& +\frac{i}{12} \sum_{i \neq j} R_{i j}\left[8\left(\partial \Gamma_{i j}\right)\left(\Gamma^{2}\right)_{j i}+\left(F_{j i}^{\alpha \beta}+9 \partial^{\alpha} \Gamma_{j i}^{\beta}\right) L_{+i j}^{\beta \alpha}\right. \\
& \left.+\Gamma_{j i}^{\alpha} \Gamma_{i j}^{\beta} F_{j j}^{\alpha \beta}\right]+\frac{i}{8} \sum_{i \neq j} S_{i j}\left(\Gamma_{i i}^{\alpha}-\Gamma_{j j}^{\alpha}\right) \\
& \times\left[\left(\Gamma_{i j}^{\alpha} \stackrel{\leftrightarrow}{\partial^{\beta}} \Gamma_{j i}^{\beta}\right)-\left(\Gamma_{j i}^{\alpha} \stackrel{\leftrightarrow}{\partial^{\beta}} \Gamma_{i j}^{\beta}\right)+\left(\Gamma_{i j}^{\beta} \stackrel{\leftrightarrow}{\partial^{\alpha}} \Gamma_{j i}^{\beta}\right)\right]
\end{aligned}
$$

which represents the effective three-particle vertices describing the local interaction of vector and axial-vector fields.

The term without derivatives $\Omega^{(0)}$, which is a sum of the terms $\propto \Gamma^{4}$, we represent in the form

$$
\Omega^{(0)}=\Omega_{1}^{(0)}+\Omega_{2}^{(0)},
$$

where $\Omega_{1}^{(0)}$ is given by Eq. (91), and

$$
\begin{aligned}
\Omega_{2}^{(0)}= & \sum_{i \neq j \neq k} R_{i j}\left[\left(L_{-}^{\alpha \alpha}\right)_{j i}\left(\Gamma_{i k} \Gamma_{k j}\right)+\left(\Gamma_{j i} \Gamma_{i j}\right)\left(\Gamma_{j k} \Gamma_{k j}\right)\right] \\
& +\frac{1}{3} \delta_{\alpha \beta \gamma \sigma} \sum_{i \neq j} S_{i j}\left(\Gamma_{i i}^{\alpha} \Gamma_{i i}^{\beta} \Gamma_{i j}^{\gamma} \Gamma_{j i}^{\sigma}-\Gamma_{i i}^{\alpha} \Gamma_{i j}^{\beta} \Gamma_{j j}^{\gamma} \Gamma_{j i}^{\sigma}\right. \\
& \left.-\frac{1}{2} \Gamma_{i j}^{\alpha} \Gamma_{j i}^{\beta} \Gamma_{i j}^{\gamma} \Gamma_{j i}^{\sigma}\right) .
\end{aligned}
$$

Here, the expression in the parentheses $\left(\Gamma_{i j} \Gamma_{j i}\right)$ is understood to be summed over alpha $\Gamma_{i j}^{\alpha} \Gamma_{j i}^{\alpha}$.

\section{CONTINUATION TO MINKOWSKI SPACE}

Let us write the Lagrangian of the effective theory arising from the considered 1/ $M$ expansion (28) after continuation to Minkowski space-time

$$
\mathcal{L}_{\text {eff }}=-\left.\frac{N_{c}}{32 \pi^{2}} \sum_{n=1}^{\infty} \operatorname{tr}_{D} b_{n}(x, x)\right|_{M} .
$$

The index $M$ explicitly indicates that coefficients $b_{n}$ belong to Minkowski space. The rules of such continuation are given in Appendix A. 
The coefficient $b_{1}(x, x)$ given by Eq. (38) in Minkowski space-time has the following form:

$$
\left.b_{1}\right|_{M}=\operatorname{tr}_{f}\left[J_{0} \circ(-Y)-\frac{1}{4}\left(\Delta J_{0} \circ \Gamma^{\mu}\right) \Gamma_{\mu}\right] .
$$

In particular, $\Gamma^{\mu}=v^{\mu}+\gamma^{5} a^{\mu}$, and

$$
\begin{aligned}
Y= & s^{2}-\{s, M\}+p^{2}+i \gamma^{5}[s-M, p] \\
& -\frac{i}{4}\left[\gamma^{\mu}, \gamma^{\nu}\right]\left(v_{\mu \nu}+\gamma^{5} a_{\mu \nu}\right)-i \gamma^{\mu}\left(\nabla_{\mu} s+i \gamma^{5} \nabla_{\mu} p\right),
\end{aligned}
$$

where

$$
\begin{gathered}
v_{\mu \nu}=\partial_{\mu} v_{\nu}-\partial_{\nu} v_{\mu}-i\left[v_{\mu}, v_{\nu}\right]-i\left[a_{\mu}, a_{\nu}\right], \\
a_{\mu \nu}=\partial_{\mu} a_{\nu}-\partial_{\nu} a_{\mu}-i\left[v_{\mu}, a_{\nu}\right]-i\left[a_{\mu}, v_{\nu}\right], \\
\nabla_{\mu} s=\partial_{\mu} s-i\left[v_{\mu}, s-M\right]-\left\{a_{\mu}, p\right\},
\end{gathered}
$$

$$
\nabla_{\mu} p=\partial_{\mu} p-i\left[v_{\mu}, p\right]+\left\{a_{\mu}, s-M\right\} .
$$

The coefficient $b_{2}(x, x)$ in Minkowski space-time can be written as

$$
\left.b_{2}\right|_{M}=\frac{1}{2} \operatorname{tr}_{f}\left[Y(J \circ Y)-\frac{1}{6} \Gamma^{\mu \nu}\left(J \circ \Gamma_{\mu \nu}\right)\right]+\Delta b_{2},
$$

where

$$
\Gamma^{\mu \nu}=\partial^{\mu} \Gamma^{\nu}-\partial^{\nu} \Gamma^{\mu}-i\left[\Gamma^{\mu}, \Gamma^{\nu}\right]
$$

is a representation of (64) in Minkowski space-time. For the contributions to $\Delta b_{2}$ we have

$$
\left.b_{2}^{(1)}\right|_{M}=\operatorname{tr}_{f}\left[N Y-\frac{i}{3}\left(R \circ \Gamma^{\mu}\right) \stackrel{\leftrightarrow}{\partial_{\mu}} Y\right] .
$$

In a similar way we find

$$
\Omega_{\left.\right|_{M}}^{(2)}=\frac{1}{24} \operatorname{tr}_{f}\left[F^{\mu \nu}\left(S \circ F_{\mu \nu}\right)+6(\partial \Gamma)(S \circ \partial \Gamma)\right],
$$

where $(\partial \Gamma)$ is understood now as $\partial_{\mu} \Gamma^{\mu}$, and

$$
\begin{aligned}
\Omega_{\left.\right|_{M} ^{(1)}=} & -\frac{i}{3} \operatorname{tr}_{f}\left[H_{\mu \nu} F^{\mu \nu}+\frac{1}{2} g_{\mu \nu \tilde{\mu} \tilde{\nu}}\left(K \circ \partial^{\mu} \Gamma^{\nu}\right) E^{\tilde{\mu} \tilde{\nu}}\right]+\frac{i}{12} \sum_{i \neq j} R_{i j}\left[8\left(\partial \Gamma_{i j}\right)\left(\Gamma^{2}\right)_{j i}-\left(F_{j i}^{\mu \nu}+9 \partial^{\mu} \Gamma_{j i}^{\nu}\right) L_{+i j}^{\nu \mu}-\Gamma_{j i}^{\mu} \Gamma_{i j}^{\nu} F_{j j}^{\mu \nu}\right] \\
& -\frac{i}{8} \sum_{i \neq j} S_{i j}\left(\Gamma_{i i}^{\mu}-\Gamma_{j j}^{\mu}\right)\left[\left(\Gamma_{i j}^{\mu} \stackrel{\leftrightarrow}{\partial^{\nu}} \Gamma_{j i}^{\nu}\right)-\left(\Gamma_{j i}^{\mu} \stackrel{\leftrightarrow}{\partial^{\nu}} \Gamma_{i j}^{\nu}\right)+\left(\Gamma_{i j}^{\nu} \stackrel{\leftrightarrow}{\partial^{\mu}} \Gamma_{j i}^{\nu}\right)\right],
\end{aligned}
$$

where $g_{\mu \nu \tilde{\mu} \tilde{\nu}}=g_{\mu \nu} g_{\tilde{\mu} \tilde{\nu}}+g_{\mu \tilde{\mu}} g_{\nu \tilde{\nu}}+g_{\mu \tilde{\nu}} g_{\nu \tilde{\mu}}$. We will not write out here an expression for $\Omega^{(0)}$, which contains the terms $\propto \Gamma^{4}$, since here, to go to the Minkowski space, it is sufficient to replace the indices, while the sign in front of any term remains unchanged.

\section{CONCLUSIONS}

Above, a new method for obtaining the effective lowenergy Lagrangian in a theory with heavy particles of unequal masses and a nontrivial flavor symmetry group has been presented in full detail. Unlike [22], where authors use the modified DeWitt WKB form and solve recurrent Schrödinger-like equations to determine the heat coefficients, our calculations are based on the formula (1), which allows us to resum the proper-time series in accordance with the contributions of corresponding one-loop Feynman diagrams with the required number of external fields. As a result, we arrive at an effective action (28), in which each of the heat coefficients can be calculated independently, and corresponds to a certain order in $1 / M^{2}$ expansion. We have explicitly calculated two leading coefficients $b_{1}(x, x)$ and $b_{2}(x, x)$ for the case of the broken $U(3) \times U(3)$ chiral symmetry. The result can be used to study the consequences of $S U(3)$ symmetry violations, for instance, in the framework of the NJL-type models, where, until now, rather rough approximations are used to describe this important effect. Although we have limited ourselves to a particular example, the proposed approach can be easily extended to the case of an arbitrary internal symmetry group, for example, to study an explicit and spontaneous symmetry violation within the context of effective field theories beyond the Standard Model.

\section{ACKNOWLEDGMENTS}

I am grateful to Professor B. Hiller for the interest in the subject and useful discussions. This work is supported by a Grant from Fundação para a Ciência e Tecnologia (FCT) through Grant No. CERN/FIS-COM/0035/2019, and the European Cooperation in Science and Technology organization through the COST Action CA16201 program.

\section{APPENDIX A: THE WICK ROTATION TO THE EUCLIDEAN SPACE-TIME}

In this Appendix, we collect some useful formulas and conventions made to perform a Wick rotation of the time axis to transit from Minkowski space-time with the metric tensor $g_{\mu \nu}=\operatorname{diag}(1,-1,-1,-1)$ to Euclidean space with metric $\delta_{\alpha \beta}=\operatorname{diag}(1,1,1,1)$ and back. Here the first two 
letters of the Greek alphabet $\alpha, \beta$ are used to enumerate the coordinates in Euclidean space $\alpha, \beta=1,2,3,4$; on the contrary, the letters $\mu, \nu$, run over the set $\mu, \nu=0,1,2,3$ in Minkowski space-time.

For coordinates $x^{\mu}$ and the Lorentz four-vector $v^{\mu}$ we apply the following correspondence rules with the Euclidean coordinates $x_{\alpha}$ and the vector components $v_{\alpha}$ :

$$
\begin{aligned}
& x^{\mu}=\left(x^{0}, \vec{x}\right) \rightarrow x_{\alpha}=\left(\vec{x}, x_{4}\right), \quad x^{0} \rightarrow-i x_{4} . \\
& \partial_{\mu}=\left(\partial_{0}, \vec{\nabla}\right) \rightarrow \partial_{\alpha}=\left(\vec{\nabla}, \partial_{4}\right), \quad \partial_{0} \rightarrow i \partial_{4} . \\
& v^{\mu}=\left(v^{0}, \vec{v}\right) \rightarrow v_{\alpha}=\left(\vec{v}, v_{4}\right), \quad v^{0} \rightarrow-i v_{4} .
\end{aligned}
$$

It follows then from (A2) and (A3) that

$$
\begin{gathered}
v^{\mu} v_{\mu}=v_{0}^{2}-\vec{v}^{2} \rightarrow-v_{4}^{2}-\vec{v}^{2}=-v_{\alpha}^{2}, \\
v^{\mu} \partial_{\mu}=v_{0} \partial_{0}+\vec{v} \vec{\nabla} \rightarrow v_{\alpha} \partial_{\alpha} .
\end{gathered}
$$

For the Dirac $\gamma$-matrices we use the following conventions:

$$
\gamma^{\mu}=\left(\gamma^{0}, \vec{\gamma}\right) \rightarrow \gamma_{\alpha}=\left(\vec{\gamma}, \gamma_{4}\right), \quad \gamma^{0} \rightarrow-i \gamma_{4} .
$$

Our choice corresponds to the case when $O(4)$ gamma matrices $\gamma_{\alpha}$ in the four-dimensional Euclidean space are anti-Hermitian. Indeed, from the standard property $\gamma^{\mu \dagger}=$ $\left(\gamma^{0},-\vec{\gamma}\right)$ and definitions made above, one finds that $\gamma_{\alpha}^{\dagger}=-\gamma_{\alpha}$. As a consequence, the Euclidean Clifford algebra emerging from $\left\{\gamma^{\mu}, \gamma^{\nu}\right\}=2 g^{\mu \nu}$ necessitates a change in the defining equation $\left\{\gamma_{\alpha}, \gamma_{\beta}\right\}=-2 \delta_{\alpha \beta}$. We have also that $\gamma^{\mu} \partial_{\mu} \rightarrow \gamma_{\alpha} \partial_{\alpha}, \gamma^{\mu} v_{\mu} \rightarrow-\gamma_{\alpha} v_{\alpha}$.

The other consequence is that $\gamma^{5}=-i \gamma^{0} \gamma^{1} \gamma^{2} \gamma^{3}$ matrix can be defined in Euclidean space as $\gamma_{5 E}=\gamma_{1} \gamma_{2} \gamma_{3} \gamma_{4}$. It follows then that $\gamma^{5} \rightarrow \gamma_{5 E}$, and both matrices are Hermitian.

For the Dirac operator

$$
D=i \gamma^{\mu} d_{\mu}-M+s+i \gamma^{5} p,
$$

where $d_{\mu}=\partial_{\mu}-i \Gamma_{\mu}$, and $\Gamma_{\mu}=v_{\mu}+\gamma^{5} a_{\mu}$, one finds

$$
D \rightarrow D_{E}=i \gamma_{\alpha} d_{\alpha}-M+s+i \gamma_{5 E} p,
$$

where $d_{\alpha}=\partial_{\alpha}+i \Gamma_{\alpha}, \Gamma_{\alpha}=v_{\alpha}+\gamma_{5 E} a_{\alpha}$.

This yields

$$
\begin{aligned}
D_{E}^{\dagger} D_{E}= & -d_{\alpha}^{2}+(s-M)^{2}+p^{2}+i \gamma_{5 E}[s-M, p] \\
& +\frac{1}{4}\left[\gamma_{\alpha}, \gamma_{\beta}\right]\left[d_{\alpha}, d_{\beta}\right]+i\left[\gamma_{\alpha}\left(s+i \gamma_{5 E} p-M\right), d_{\alpha}\right] \\
= & M^{2}-d^{2}+Y
\end{aligned}
$$

where $d^{2}=d_{\alpha} d_{\alpha}$,

$$
\begin{aligned}
Y= & s^{2}-\{s, M\}+p^{2}+i \gamma_{5 E}[s-M, p] \\
& +\frac{i}{4}\left[\gamma_{\alpha}, \gamma_{\beta}\right]\left(v_{\alpha \beta}+\gamma_{5 E} a_{\alpha \beta}\right) \\
& -i \gamma_{\alpha}\left(\nabla_{\alpha} s+i \gamma_{5 E} \nabla_{\alpha} p\right)
\end{aligned}
$$

and the chiral-covariant objects are

$$
\begin{array}{r}
v_{\alpha \beta}=\partial_{\alpha} v_{\beta}-\partial_{\beta} v_{\alpha}+i\left[v_{\alpha}, v_{\beta}\right]+i\left[a_{\alpha}, a_{\beta}\right], \\
a_{\alpha \beta}=\partial_{\alpha} a_{\beta}-\partial_{\beta} a_{\alpha}+i\left[v_{\alpha}, a_{\beta}\right]+i\left[a_{\alpha}, v_{\beta}\right], \\
\nabla_{\alpha} s=\partial_{\alpha} s+i\left[v_{\alpha}, s-M\right]+\left\{a_{\alpha}, p\right\}, \\
\nabla_{\alpha} p=\partial_{\alpha} p+i\left[v_{\alpha}, p\right]-\left\{a_{\alpha}, s-M\right\} .
\end{array}
$$

We have given these well-known formulas here both for the completeness of our presentation and to clarify notations used in the main text of our work.

\section{APPENDIX B: THE MODULUS OF THE DETERMINANT}

This is a summary of the standard steps to evaluate the modulus of determinant (3). The self-adjoint elliptic operator $\Delta=D_{E}^{\dagger} D_{E}$ can be treated as an operator acting on a fictitious Hilbert space, where the quantum-mechanical algebra of coordinates and momenta is realized, i.e., where $|x\rangle$ is an eigenvector of a commuting set of Hermitian operators $\hat{x}_{\alpha}$ such that $\hat{x}_{\alpha}|x\rangle=x_{\alpha}|x\rangle$ and $\langle x \mid y\rangle=\delta(x-y)$. The Hermitian operators, $\hat{p}_{\alpha}=-i \partial_{\alpha}$, which are conjugate to $\hat{x}_{\alpha}$, obey canonical commutation relations: $\left[\hat{x}_{\alpha}, \hat{p}_{\beta}\right]=$ $i \delta_{\alpha \beta},\left[\hat{x}_{\alpha}, \hat{x}_{\beta}\right]=\left[\hat{p}_{\alpha}, \hat{p}_{\beta}\right]=0$.

Let us consider an eigenket $|p\rangle$ of $\hat{p}_{\alpha}$. Its representative in the Schrödinger picture is $\langle x \mid p\rangle=(2 \pi)^{-2} \exp \left(i x_{\alpha} p_{\alpha}\right)$. Using this plane-wave basis one can describe the action of the operator $e^{-t \Delta}$ on the Hilbert space by the heat kernel function of $\Delta$ :

$h(x, y ; t)=\left\langle x\left|e^{-t \Delta}\right| y\right\rangle=\int d^{4} p\left\langle x\left|e^{-t \Delta}\right| p\right\rangle\langle p \mid y\rangle$.

Taking into account the relations $\left\langle x\left|\hat{p}_{\alpha}\right| p\right\rangle=p_{\alpha}\langle x \mid p\rangle=$ $-i \partial_{\alpha}^{x}\langle x \mid p\rangle$ and $\left\langle x\left|\hat{x}_{\alpha}\right| p\right\rangle=x_{\alpha}\langle x \mid p\rangle$, one can proceed:

$$
\begin{aligned}
\left\langle x\left|e^{-t \Delta}\right| y\right\rangle & =\int d^{4} p e^{-t \Delta_{x}}\langle x \mid p\rangle\langle p \mid y\rangle \\
& =\int \frac{d^{4} p}{(2 \pi)^{4}} e^{-i p y} e^{-t \Delta_{x}} e^{i p x} \\
& =\int \frac{d^{4} p}{(2 \pi)^{4}} e^{i p(x-y)}\left(e^{-i p x} e^{-t \Delta_{x}} e^{i p x}\right),
\end{aligned}
$$

where $\Delta_{x}$ is the operator $\Delta$ in the coordinate representation. 
Now note that

$$
\begin{aligned}
e^{-i p x} e^{-t \Delta_{x}} e^{i p x} & =\sum_{n=0}^{\infty} \frac{(-t)^{n}}{n !} e^{-i p x}\left(\Delta_{x}\right)^{n} e^{i p x} \\
& =\sum_{n=0}^{\infty} \frac{(-t)^{n}}{n !}\left(e^{-i p x} \Delta_{x} e^{i p x}\right)^{n},
\end{aligned}
$$

where

$$
\begin{aligned}
e^{-i p x} \Delta_{x} e^{i p x} & =\Delta_{x}-\left[i p x, \Delta_{x}\right]+\frac{1}{2 !}\left[i p x,\left[i p x, \Delta_{x}\right]\right] \\
& =\Delta_{x}-2 i p_{\alpha} d_{\alpha}+p^{2}
\end{aligned}
$$

Since the operator $\Delta_{x}$ contains derivatives of at most second order, its higher-order commutators with ipx are equal to zero. At the last stage we used Eq. (A9), namely

$$
\begin{aligned}
{\left[i p x, \Delta_{x}\right] } & =\left[i p x, M^{2}-d^{2}+Y\right]=-\left[i p x, d^{2}\right] \\
& =-\left[i p x, \partial_{\alpha}^{2}+2 i \Gamma_{\alpha} \partial_{\alpha}\right] \\
& =-\left[i p x, \partial_{\alpha}\right] \partial_{\alpha}-\partial_{\alpha}\left[i p x, \partial_{\alpha}\right]-2 i \Gamma_{\alpha}\left[i p x, \partial_{\alpha}\right] \\
& =2 i p_{\alpha} d_{\alpha}, \\
{\left[i p x,\left[i p x, \Delta_{x}\right]\right] } & =\left[i p x, 2 i p_{\alpha} d_{\alpha}\right]=2 p_{\alpha}^{2} .
\end{aligned}
$$

As a result we obtain

$$
\begin{aligned}
\left\langle x\left|e^{-t \Delta}\right| y\right\rangle & =\int \frac{d^{4} p}{(2 \pi)^{4}} e^{i p(x-y)} e^{-t p^{2}} e^{-t\left(\Delta_{x}-2 i p d\right)} \\
& =\int \frac{d^{4} p}{\left(4 \pi^{2} t\right)^{2}} e^{-p^{2}+\frac{i}{\sqrt{t}} p(x-y)} e^{-t\left(\Delta_{x}-\frac{2 i p d}{\sqrt{t}}\right)},
\end{aligned}
$$

or, for the trace

$$
\begin{aligned}
\operatorname{Tr}\left(e^{-t \Delta}\right) & =\int d^{4} x \operatorname{tr}_{I}\left\langle x\left|e^{-t \Delta}\right| x\right\rangle \\
& =\int \frac{d^{4} x d^{4} p}{\left(4 \pi^{2} t\right)^{2}} e^{-p^{2}} \operatorname{tr}_{I} e^{-t\left(\Delta_{x}-\frac{2 i p d}{\sqrt{t}}\right)}
\end{aligned}
$$

where $\operatorname{tr}_{I}$ means the trace over color, isospin, and Dirac indices. Expanding the last exponent in the series in the proper time, one can take the integrals over four-momenta with the help of the formulas

$$
\int \frac{d^{4} p}{(2 \pi)^{4}} e^{-p^{2}} p_{\alpha_{1}} p_{\alpha_{2}} \ldots p_{\alpha_{2 n}}=\frac{\delta_{\alpha_{1} \alpha_{2} \ldots \alpha_{2 n}}}{2^{n}(4 \pi)^{2}}
$$

Note that only the even number of internal momenta $p_{\alpha}$ contribute; the odd number, obviously, gives zero. The totally symmetric tensor $\delta_{\alpha_{1} \alpha_{2} \ldots \alpha_{2 n}}$ is a product of Kronecker's deltas $\delta_{\alpha_{1} \alpha_{2}} \delta_{\alpha_{3} \alpha_{4}} \ldots \delta_{\alpha_{2 n-1} \alpha_{2 n}}$ plus all possible permutations of indices; there are $(2 n-1)$ !! terms in total. For example,

$$
\delta_{\alpha_{1} \alpha_{2} \alpha_{3} \alpha_{4}}=\delta_{\alpha_{1}}^{\alpha_{2}} \delta_{\alpha_{4}}^{\alpha_{3}}+\delta_{\alpha_{1}}^{\alpha_{3}} \delta_{\alpha_{4}}^{\alpha_{2}}+\delta_{\alpha_{1}}^{\alpha_{4}} \delta_{\alpha_{2}}^{\alpha_{3}} .
$$

Finally, combining (3) and (B6) we come to Eq. (14).

\section{APPENDIX C: PROOF OF THE FORMULA (22)}

Here we present some details related to obtaining of Eq. (22). Let us first consider the case when $n=1$,

$$
\begin{aligned}
\operatorname{tr}_{f} & {\left[e^{-t M^{2}} f_{1}(t, A)\right] } \\
& =\sum_{i, j, k}^{3} e^{-t m_{i}^{2}} \int_{0}^{t} d s e^{s\left(m_{j}^{2}-m_{k}^{2}\right)} \operatorname{tr}_{f}\left(E_{i} E_{j} A E_{k}\right) \\
& =\sum_{i} t e^{-t m_{i}^{2}} \operatorname{tr}_{f} A_{i} .
\end{aligned}
$$

Hence,

$$
c_{i}(t)=e^{-t m_{i}^{2}} .
$$

For $n=2$ we have

$$
\begin{aligned}
\operatorname{tr}_{f}\left[e^{-t M^{2}} f_{2}(t, A)\right]= & \sum_{i, j}^{3} \int_{0}^{t} d s_{1} \int_{0}^{s_{1}} d s_{2} \\
& \times e^{\left(s_{1}-s_{2}-t\right) m_{i}^{2}} e^{\left(s_{2}-s_{1}\right) m_{j}^{2}} \operatorname{tr}_{f}\left(A_{i} A_{j}\right) \\
& =\sum_{i, j}^{3}\left(t \frac{e^{-t m_{i}^{2}}}{\Delta_{j i}}+\frac{e^{-t m_{j}^{2}}-e^{-t m_{i}^{2}}}{\Delta_{i j}^{2}}\right) \operatorname{tr}_{f}\left(A_{i} A_{j}\right) \\
& =\sum_{i, j}^{3} \frac{t}{2 \Delta_{j i}}\left(e^{-t m_{i}^{2}}-e^{-t m_{j}^{2}}\right) \operatorname{tr}_{f}\left(A_{i} A_{j}\right) .
\end{aligned}
$$

Here $\Delta_{i j}=m_{i}^{2}-m_{j}^{2}$, and in the last stage we used that trace is symmetric under an exchange $i \leftrightarrow j$. It gives

$$
c_{i_{1} i_{2}}(t)=\frac{1}{t} \sum_{\text {perm }} \frac{c_{i_{1}}(t)}{\Delta_{i_{2} i_{1}}} .
$$

The sum includes two terms obtained by cyclically permuting the indices $\left(i_{1}, i_{2}\right) \rightarrow\left(i_{2}, i_{1}\right)$.

For $n=3$ 


$$
\begin{aligned}
\operatorname{tr}_{f}\left[e^{-t M^{2}} f_{3}(t, A)\right] & =\sum_{i, j, k}^{3} \int_{0}^{t} d s_{1} \int_{0}^{s_{1}} d s_{2} \int_{0}^{s_{2}} d s_{3} e^{\left(s_{1}-s_{3}-t\right) m_{i}^{2}} e^{\left(s_{2}-s_{1}\right) m_{j}^{2}} e^{\left(s_{3}-s_{2}\right) m_{k}^{2} \operatorname{tr}_{f}\left(A_{i} A_{j} A_{k}\right)} \\
& =\sum_{i, j, k}^{3}\left(\frac{t c_{i}(t)}{\Delta_{i j} \Delta_{i k}}+\frac{c_{i}(t)-c_{j}(t)}{\Delta_{i j}^{2} \Delta_{j k}}+\frac{c_{i}(t)-c_{k}(t)}{\Delta_{i k}^{2} \Delta_{k j}}\right) \operatorname{tr}_{f}\left(A_{i} A_{j} A_{k}\right) .
\end{aligned}
$$

Since the trace is symmetric with respect to the cyclic permutation of matrices, one can add to this expression two others, obtained by cyclic permutations of indices $(i, j, k) \rightarrow(j, k, i)$ and $(i, j, k) \rightarrow(k, i, j)$, and the result should be divided by three. As a result, only the first term in parentheses will lead to the nonzero contribution. This yields the following expression for the coefficient:

$$
c_{i_{1} i_{2} i_{3}}(t)=\frac{2 !}{t^{2}} \sum_{\text {perm }} \frac{c_{i_{1}}(t)}{\Delta_{i_{2} i_{1}} \Delta_{i_{3} i_{1}}} .
$$

Consider the case $n=4$. Here we have

$$
\begin{aligned}
\operatorname{tr}_{f}\left[e^{-t M^{2}} f_{4}(t, A)\right] & =\sum_{i, j, k, l}^{3} \int_{0}^{t} d s_{1} \int_{0}^{s_{1}} d s_{2} \int_{0}^{s_{2}} d s_{3} \int_{0}^{s_{3}} d s_{4} e^{\left(s_{1}-s_{4}-t\right) m_{i}^{2}} e^{\left(s_{2}-s_{1}\right) m_{j}^{2}} e^{\left(s_{3}-s_{2}\right) m_{k}^{2}} e^{\left(s_{4}-s_{3}\right) m_{l}^{2}} \operatorname{tr}_{f}\left(A_{i} A_{j} A_{k} A_{l}\right) \\
& =\sum_{i, j, k, l}^{3}\left(\frac{t c_{i}(t)}{\Delta_{j i} \Delta_{k i} \Delta_{l i}}+\frac{c_{j}(t)-c_{i}(t)}{\Delta_{i j}^{2} \Delta_{k j} \Delta_{l j}}+\frac{c_{k}(t)-c_{i}(t)}{\Delta_{i k}^{2} \Delta_{l k} \Delta_{j k}}+\frac{c_{l}(t)-c_{i}(t)}{\Delta_{i l}^{2} \Delta_{k l} \Delta_{j l}}\right) \operatorname{tr}_{f}\left(A_{i} A_{j} A_{k} A_{l}\right) .
\end{aligned}
$$

By using again the cyclic permutation property of trace, one can easily find out that only the first term in parentheses will contribute with a nonzero result. Here we have four cyclic permutations of indices; correspondingly it gives four terms for the coefficient

$$
c_{i_{1} i_{2} i_{3} i_{4}}(t)=\frac{3 !}{t^{3}} \sum_{\text {perm }} \frac{c_{i_{1}}(t)}{\Delta_{i_{2} i_{1}} \Delta_{i_{3} i_{1}} \Delta_{i_{4} i_{1}}} .
$$

Continuing the above procedure, we can find by induction an arbitrary coefficient in formula (22):

$$
c_{i_{1} i_{2} \ldots i_{n}}(t)=\frac{(n-1) !}{t^{n-1}} \sum_{p e r m} \frac{c_{i_{1}}(t)}{\Delta_{i_{2} i_{1}} \Delta_{i_{3} i_{1}} \ldots \Delta_{i_{n} i_{1}}} .
$$

Although the sum includes $n$ terms obtained by cyclically permuting the indices $i_{1}, i_{2}, \ldots, i_{n}$, it is easy to show that the coefficients $c_{i_{1} i_{2} \ldots i_{n}}$ are totally symmetric.

One can also establish a recurrence formula relating coefficients of order $n, c_{i_{1} i_{2} \ldots i_{n}}(t)$, for $n>1$, with coefficients of order $n-1$ :

$c_{i_{1} i_{2} \ldots i_{n}}(t)=\frac{n-1}{t \Delta_{i_{n-1} i_{n}}}\left[c_{i_{1} i_{2} \ldots i_{n-2} i_{n}}(t)-c_{i_{1} i_{2} \ldots i_{n-1}}(t)\right]$.

This relation follows from the following easily verified identities:

$$
\begin{gathered}
\sum_{\text {perm }} \frac{1}{\Delta_{i_{2} i_{1}}}=0, \\
\sum_{\text {perm }} \frac{1}{\Delta_{i_{2} i_{1}} \Delta_{i_{3} i_{1}}}=0,
\end{gathered}
$$

$$
\sum_{\text {perm }} \frac{1}{\Delta_{i_{2} i_{1}} \Delta_{i_{3} i_{1}} \ldots \Delta_{i_{n} i_{1}}}=0 .
$$

Recurrent formula (C10), inter alia, simplifies the proof of the relations

$$
c_{i}(t)=c_{i i}(t)=c_{i i \ldots i}(t)
$$

Indeed, one has, for instance,

$$
\begin{aligned}
c_{i i}(t) & =\lim _{m_{i} \rightarrow m_{j}} c_{i j}(t)=\lim _{m_{i} \rightarrow m_{j}} \frac{e^{-t m_{j}^{2}}-e^{-t m_{i}^{2}}}{t \Delta_{i j}} \\
& =\lim _{m_{i} \rightarrow m_{j}} e^{-t m_{i}^{2}} \frac{e^{t \Delta_{i j}}-1}{t \Delta_{i j}}=c_{i}(t) .
\end{aligned}
$$

Using (C10) we can extend this to any $c_{i i \ldots i}(t)$ case. 


\section{APPENDIX D: INTEGRALS OVER PROPER TIME}

If the masses of the Fermi fields are nondegenerate, integration over the proper time leads to expressions depending on the specific masses of the constituent quarks in the corresponding Feynman diagram. Accordingly, the number of different loop integrals increases. We will classify the integrals in accord with the number of internal quark lines presented in the loop diagram. Since we are dealing with a $1 / M$ expansion, the integrals presented below give only the leading terms of this expansion. Along with separating the main divergences of the Feynman diagrams, such integrals contain finite contributions that vanish in the case of equal quark masses. These finite contributions are uniquely determined by the coefficients $c_{i_{1} i_{2} \ldots i_{n}}$ that are completely symmetric with respect to the permutation of any two indices.

\section{Tadpole diagrams}

The diagrams with only one internal quark line (tadpole) are associated with the proper-time integral:

$$
\int \frac{d t}{t^{2}} \rho_{t, \Lambda} c_{i}(t)=J_{0}\left(m_{i}^{2}\right) .
$$

We will use Pauli-Villars regularization corresponding to two subtractions at the mass-scale $\Lambda$ :

$$
\rho_{t, \Lambda}=1-\left(1+t \Lambda^{2}\right) e^{-t \Lambda^{2}} .
$$

In this case, it follows that

$$
J_{0}\left(m_{i}^{2}\right)=\Lambda^{2}-m_{i}^{2} \ln \left(1+\frac{\Lambda^{2}}{m_{i}^{2}}\right) .
$$

This obviously coincides with (19).

\section{Self-energy diagrams}

The quark-loop self-energy graphs are described by the proper-time integrals:

$$
\int \frac{d t}{t^{2-n}} \rho_{t, \Lambda} c_{i j}(t)=J_{n}\left(m_{i}^{2}, m_{j}^{2}\right)
$$

Due to the symmetry of the coefficients $c_{i j}=c_{j i}$ the result of integration is symmetric with respect to the replacement $m_{i} \leftrightarrow m_{j}$. Indeed, in the case $n=0$, we have

$$
\begin{aligned}
J_{0}\left(m_{i}^{2}, m_{j}^{2}\right)= & \frac{\Lambda^{2}}{2}+\frac{\Lambda^{4}}{2 \Delta_{i j}} \ln \frac{\Lambda^{2}+m_{i}^{2}}{\Lambda^{2}+m_{j}^{2}} \\
& -\frac{1}{2 \Delta_{i j}}\left[m_{i}^{4} \ln \left(1+\frac{\Lambda^{2}}{m_{i}^{2}}\right)-m_{j}^{4} \ln \left(1+\frac{\Lambda^{2}}{m_{j}^{2}}\right)\right],
\end{aligned}
$$

where $\Delta_{i j}=m_{i}^{2}-m_{j}^{2}$. In the coincidence limit $m_{i} \rightarrow m_{j}$,

$$
\lim _{m_{i} \rightarrow m_{j}} J_{0}\left(m_{i}^{2}, m_{j}^{2}\right)=J_{0}\left(m_{i}^{2}\right) .
$$

The other integral for $n=1$ is given by the difference of the tadpole contributions:

$$
\begin{aligned}
J_{1}\left(m_{i}^{2}, m_{j}^{2}\right) & =\frac{1}{\Delta_{i j}}\left[J_{0}\left(m_{j}^{2}\right)-J_{0}\left(m_{i}^{2}\right)\right] \\
& =\frac{1}{\Delta_{i j}}\left[m_{i}^{2} \ln \left(1+\frac{\Lambda^{2}}{m_{i}^{2}}\right)-m_{j}^{2} \ln \left(1+\frac{\Lambda^{2}}{m_{j}^{2}}\right)\right] .
\end{aligned}
$$

Since we also have expressions for the similar $J_{1}$-type contributions coming from the triangle and box diagrams, we will collect them together in one formula:

$$
\left(\begin{array}{c}
J_{i j} \\
J_{i j k} \\
J_{i i j k}
\end{array}\right) \equiv \int_{0}^{\infty} \frac{d t}{t} \rho_{t, \Lambda}\left(\begin{array}{c}
c_{i j}(t) \\
c_{i j k}(t) \\
c_{i i j k}(t)
\end{array}\right) .
$$

From Eq. (C14), it follows that

$$
J_{i i}=J_{i i i}=J_{i i i i}=J_{1}\left(m_{i}^{2}\right) \equiv J_{i} .
$$

The integrals take this form when all quark masses in the loop are equal.

\section{Triangle diagrams}

The proper-time integral

$$
\int \frac{d t}{t} \rho_{t, \Lambda} c_{i j k}(t)=J_{1}\left(m_{i}^{2}, m_{j}^{2}, m_{k}^{2}\right) \equiv J_{i j k}
$$

describes a local part of the diagram with three internal quark lines. As before, the full symmetry of the coefficient $c_{i j k}$ allows one to conclude that this integral does not change when any two fermion masses are exchanged. To verify this, we use the formula (C10), which at the first stage of calculations leads to a seemingly asymmetric result:

$$
J_{i j k}=\frac{2}{\Delta_{j k}}\left[J_{0}\left(m_{i}^{2}, m_{k}^{2}\right)-J_{0}\left(m_{i}^{2}, m_{j}^{2}\right)\right]
$$

where $J_{0}\left(m_{i}^{2}, m_{j}^{2}\right)$ is given by Eq. (D5). However, the direct calculation of the right-hand side of this expression shows explicitly its total symmetry: 


$$
\begin{aligned}
J_{1}\left(m_{i}^{2}, m_{j}^{2}, m_{k}^{2}\right)= & \frac{1}{\Delta_{i j} \Delta_{j k} \Delta_{k i}}\left[\Lambda^{4}\left(m_{i}^{2} \ln \frac{\Lambda^{2}+m_{k}^{2}}{\Lambda^{2}+m_{j}^{2}}+m_{j}^{2} \ln \frac{\Lambda^{2}+m_{i}^{2}}{\Lambda^{2}+m_{k}^{2}}+m_{k}^{2} \ln \frac{\Lambda^{2}+m_{j}^{2}}{\Lambda^{2}+m_{i}^{2}}\right)\right. \\
& \left.+m_{i}^{4} \Delta_{k j} \ln \left(1+\frac{\Lambda^{2}}{m_{i}^{2}}\right)+m_{j}^{4} \Delta_{i k} \ln \left(1+\frac{\Lambda^{2}}{m_{j}^{2}}\right)+m_{k}^{4} \Delta_{j i} \ln \left(1+\frac{\Lambda^{2}}{m_{k}^{2}}\right)\right] .
\end{aligned}
$$

This expression allows us to obtain a particular value corresponding to the cases with two equal masses:

$$
\begin{aligned}
J_{1}\left(m_{i}^{2}, m_{i}^{2}, m_{j}^{2}\right) & =\frac{2}{\Delta_{i j}}\left[J_{0}\left(m_{i}^{2}, m_{j}^{2}\right)-J_{0}\left(m_{i}^{2}\right)\right] \\
& =\frac{1}{\Delta_{i j}}\left[2 m_{i}^{2} \ln \left(1+\frac{\Lambda^{2}}{m_{i}^{2}}\right)-\Lambda^{2}\right]+\frac{1}{\Delta_{i j}^{2}}\left[m_{j}^{4} \ln \left(1+\frac{\Lambda^{2}}{m_{j}^{2}}\right)-m_{i}^{4} \ln \left(1+\frac{\Lambda^{2}}{m_{i}^{2}}\right)+\Lambda^{4} \ln \frac{\Lambda^{2}+m_{i}^{2}}{\Lambda^{2}+m_{j}^{2}}\right] .
\end{aligned}
$$

It should be noted that in the theory considered, there are only three different flavor states of quarks: $u, d$, and $s$. Therefore, Eq. (D12) describes the six possible cases: uud, $u u s, d d u, d d s, s s u$, and $s s d$. Notice also that a contribution, for instance, of uus triangle differs from ssu one. The difference is antisymmetric with respect to the replacement $m_{i} \leftrightarrow m_{j}$,

$$
J_{i i j}-J_{j j i}=\frac{2}{\Delta_{i j}} \Delta J_{0}\left(m_{i}^{2}, m_{j}^{2}\right)
$$

where

$$
\Delta J_{0}\left(m_{i}^{2}, m_{j}^{2}\right)=2 J_{0}\left(m_{i}^{2}, m_{j}^{2}\right)-J_{0}\left(m_{i}^{2}\right)-J_{0}\left(m_{j}^{2}\right) .
$$

This value is finite at $\Lambda \rightarrow \infty$. The sum is

$$
J_{i i j}+J_{j j i}=2 J_{1}\left(m_{i}^{2}, m_{j}^{2}\right) .
$$

As a consequence, we have

$$
J_{1}\left(m_{i}^{2}, m_{i}^{2}, m_{j}^{2}\right)=J_{1}\left(m_{i}^{2}, m_{j}^{2}\right)+R\left(m_{i}^{2}, m_{j}^{2}\right),
$$

where the antisymmetric part of this expression is defined as

$$
R\left(m_{i}^{2}, m_{j}^{2}\right)=\frac{\Delta J_{0}\left(m_{i}^{2}, m_{j}^{2}\right)}{\Delta_{i j}} \equiv R_{i j} .
$$

\section{Box diagrams}

Consider the last of the divergent-type expressions that arise when one calculates the quark box diagrams with external meson fields. These are given by the integrals of the form

$$
\int \frac{d t}{t} \rho_{t, \Lambda} c_{i j k l}(t)=J_{1}\left(m_{i}^{2}, m_{j}^{2}, m_{k}^{2}, m_{l}^{2}\right)
$$

Since quarks have only three flavors, at least two indices in $c_{i j k l}$ coincide. Therefore, we actually need to calculate the master integral:

$$
\int \frac{d t}{t} \rho_{t, \Lambda} c_{i i j k}(t)=J_{i i j k}
$$

To this end, we again use the formula (C10), which in this case takes the form

$$
c_{i i j k}(t)=\frac{3}{t \Delta_{k j}}\left[c_{i i j}(t)-c_{i i k}(t)\right] .
$$

The quadratic divergences arising when one integrates each of the coefficients $c_{i i j}(t)$ are canceled in the difference (D20). As a result, we arrive at a logarithmically divergent (at $\Lambda \rightarrow \infty$ ) expression:

$$
\begin{aligned}
J_{i i j k}= & \frac{1}{\Delta_{i j} \Delta_{i k}}\left[3 m_{i}^{4} \ln \left(1+\frac{\Lambda^{2}}{m_{i}^{2}}\right)-\Lambda^{2}\left(2 \Lambda^{2}+m_{i}^{2}\right)\right] \\
& +\frac{1}{\Delta_{k j}}\left\{\frac{1}{\Delta_{i j}^{2}}\left[m_{i}^{6} \ln \left(1+\frac{\Lambda^{2}}{m_{i}^{2}}\right)-m_{j}^{6} \ln \left(1+\frac{\Lambda^{2}}{m_{j}^{2}}\right)+\Lambda^{4}\left(2 \Lambda^{2}+3 m_{j}^{2}\right) \ln \frac{\Lambda^{2}+m_{j}^{2}}{\Lambda^{2}+m_{i}^{2}}\right]\right. \\
& \left.-\frac{1}{\Delta_{i k}^{2}}\left[m_{i}^{6} \ln \left(1+\frac{\Lambda^{2}}{m_{i}^{2}}\right)-m_{k}^{6} \ln \left(1+\frac{\Lambda^{2}}{m_{k}^{2}}\right)+\Lambda^{4}\left(2 \Lambda^{2}+3 m_{k}^{2}\right) \ln \frac{\Lambda^{2}+m_{k}^{2}}{\Lambda^{2}+m_{i}^{2}}\right]\right\}
\end{aligned}
$$


Notice that in full accord with (D20) we have

$$
J_{i i j k}=J_{i i k j}
$$

Cases with two different types of flavors can be obtained from this expression by passing to the corresponding limits of equal masses. There are two of them: $J_{i i j j}$ and $J_{i i i j}$. As a result, we have

$$
\begin{aligned}
J_{i i j j}= & \frac{1}{\Delta_{i j}^{2}}\left\{m_{i}^{4}\left(1-2 \frac{m_{j}^{2}}{\Delta_{i j}}\right) \ln \left(1+\frac{\Lambda^{2}}{m_{i}^{2}}\right)+m_{j}^{4}\left(1-2 \frac{m_{i}^{2}}{\Delta_{j i}}\right) \ln \left(1+\frac{\Lambda^{2}}{m_{j}^{2}}\right)\right. \\
& \left.-\Lambda^{2}\left(4 \Lambda^{2}+m_{i}^{2}+m_{j}^{2}\right)-\Lambda^{4}\left[3+\frac{2}{\Delta_{i j}}\left(2 \Lambda^{2}+3 m_{j}^{2}\right)\right] \ln \frac{\Lambda^{2}+m_{j}^{2}}{\Lambda^{2}+m_{i}^{2}}\right\}, \\
J_{i i i j}= & \frac{1}{\Delta_{i j}^{3}}\left\{m_{i}^{6} \ln \left(1+\frac{\Lambda^{2}}{m_{i}^{2}}\right)-m_{j}^{6} \ln \left(1+\frac{\Lambda^{2}}{m_{j}^{2}}\right)+\Delta_{i j} \Lambda^{2}\left(2 \Lambda^{2}-m_{i}^{2}+2 m_{j}^{2}\right)\right. \\
& \left.-3 \Delta_{i j} m_{i}^{2} m_{j}^{2} \ln \left(1+\frac{\Lambda^{2}}{m_{i}^{2}}\right)+\Lambda^{4}\left(2 \Lambda^{2}+3 m_{j}^{2}\right) \ln \frac{\Lambda^{2}+m_{j}^{2}}{\Lambda^{2}+m_{i}^{2}}\right\} .
\end{aligned}
$$

From (D6) and (D23) we find the relation

$$
J_{i i j j}=J_{i j}-S\left(m_{i}^{2}, m_{j}^{2}\right)
$$

where

$S\left(m_{i}^{2}, m_{j}^{2}\right)=\frac{1}{\Delta_{i j}^{3}}\left\{m_{i}^{2} m_{j}^{2}\left(m_{i}^{2}+m_{j}^{2}\right) \ln \frac{\left(\Lambda^{2}+m_{i}^{2}\right) m_{j}^{2}}{\left(\Lambda^{2}+m_{j}^{2}\right) m_{i}^{2}}+\Lambda^{4}\left[4 \Lambda^{2}+3\left(m_{i}^{2}+m_{j}^{2}\right)\right] \ln \frac{\Lambda^{2}+m_{j}^{2}}{\Lambda^{2}+m_{i}^{2}}\right\}+\frac{\Lambda^{2}}{\Delta_{i j}^{2}}\left(4 \Lambda^{2}+m_{i}^{2}+m_{j}^{2}\right)$.

The latter expression is symmetric under exchange of masses and finite at $\Lambda \rightarrow \infty$.

Equation (D24) describes the six possible variants of the distribution of quarks along the inner lines of the box diagram; these are uиud, uиus, $d d d u, d d d s, s s s u$, and sssd. In particular, it follows that

$$
J_{i i i j}-J_{j j j i}=3 R_{i j}
$$

where $R_{i j}$ is given by (D17). On the other hand, the sum of these integrals is

$$
J_{i i i j}+J_{j j j i}=2 J_{1}\left(m_{i}^{2}, m_{j}^{2}\right)+S\left(m_{i}^{2}, m_{j}^{2}\right) .
$$

\section{APPENDIX E: MIXED SUMS FOR $b_{n}(x, x)$}

In the main text we make use summations which contain both the mass-dependent remnant, $J_{i_{1} i_{2} \ldots i_{n}}$, of one-loop quark integrals and the corresponding field-dependent coefficients $T_{i_{1} i_{2} \ldots i_{n}}$. The result of such summations depends on the properties of $J_{i_{1} i_{2} \ldots i_{n}}$ which were established in Appendix D. Here some important results on these sums are presented.
The simplest case is given by the sum

$$
\begin{aligned}
\sum_{i, j} J_{i j} T_{i j} & =\sum_{i, j} J_{i j} T_{(i j)} \\
& =\sum_{i=1}^{3} J_{i} T_{i i}+2 \sum_{i<j} J_{i j} T_{(i j)},
\end{aligned}
$$

where

$$
T_{(i j)}=\frac{1}{2}\left(T_{i j}+T_{j i}\right)
$$

Consider now the sum related to the triangle diagrams. The corresponding one-loop integral induces at leading order of its large mass expansion the function $J_{i j k}$, which is known to be completely symmetric under any exchange of masses. Due to this symmetry we have

$$
\begin{aligned}
\sum_{i, j, k} J_{i j k} T_{i j k} & =\sum_{i, j, k} J_{i j k} T_{(i j k)} \\
& =\sum_{i} J_{i} T_{i i i}+3 \sum_{i \neq j} J_{i i j} T_{(i i j)}+6 J_{123} T_{(123)},
\end{aligned}
$$


where

$T_{(i j k)}=\frac{1}{6}\left(T_{i j k}+T_{j i k}+T_{i k j}+T_{j k i}+T_{k i j}+T_{k j i}\right)$

is a totally symmetric function of quark masses. The sum has $3+3 \times 6+6=27$ terms in all, as it should be. Now we make use of Eq. (D16) to obtain

$$
\sum_{i \neq j} J_{i i j} T_{(i i j)}=\sum_{i<j}\left(J_{i j} T_{i i j}^{+}+R_{i j} T_{i i j}^{-}\right)
$$

where $T_{i i j}^{ \pm}=T_{(i i j)} \pm T_{(j j i)}$. As a result we finally have

$$
\begin{aligned}
\sum_{i, j, k} J_{i j k} T_{i j k}= & \sum_{i} J_{i} T_{i i i}+3 \sum_{i<j}\left(J_{i j} T_{i i j}^{+}+R_{i j} T_{i i j}^{-}\right) \\
& +6 J_{123} T_{(123)} .
\end{aligned}
$$

The next sum describes the contribution of the fermion box diagrams with external spin-1 fields to the coefficient $b_{2}(x, x)$. Here we have

$$
\begin{aligned}
\sum_{i, j, k, l} J_{i j k l} T_{i j k l}= & \sum_{i, j, k, l} J_{i j k l} T_{(i j k l)} \\
= & \sum_{i} J_{i} T_{i i i i}+4 \sum_{i \neq j} J_{i i i j} T_{(i i i j)}+6 \sum_{i<j} J_{i i j j} T_{(i i j j)} \\
& +12 \sum_{\substack{i \neq j \neq k \\
j<k}} J_{i i j k} T_{(i i j k)} .
\end{aligned}
$$

This formula is written for the specific case when each of the indices $i, j, k, l$ takes only three possible values 1,2 , or 3 (we consider fermion fields with three flavors), so $T_{i j k l}$ has at least two equal indices. As a result, the sum (E7) has $3+4 \times 6+6 \times 3+12 \times 3=81$ terms, and we need to know only the following coefficients:

$$
\begin{aligned}
T_{(i i i j)}= & \frac{1}{4}\left(T_{i i i j}+T_{i i j i}+T_{i j i i}+T_{j i i i}\right), \\
T_{(i i j j)}= & \frac{1}{6}\left(T_{i i j j}+T_{j j i i}+T_{i j i j}+T_{j i j i}+T_{i j j i}+T_{j i i j}\right), \\
T_{(i i j k)}= & \frac{1}{12}\left(T_{i i j k}+T_{i i k j}+T_{k i i j}+T_{j i i k}+T_{j k i i}+T_{k j i i}\right. \\
& \left.+T_{i j i k}+T_{i k i j}+T_{j i k i}+T_{k i j i}+T_{i j k i}+T_{i k j i}\right) .
\end{aligned}
$$

With the use of (D27) and (D28) one can set apart the divergent part of the proper-time integral $J_{i i i j}$ in the form $J_{i j}$, and as a result to obtain that

$$
\sum_{i \neq j} J_{i i i j} T_{(i i i j)}=\sum_{i<j}\left(J_{i j}+\frac{1}{2} S_{i j}\right) T_{i i i j}^{+}+\frac{3}{2} \sum_{i<j} R_{i j} T_{i i i j}^{-},
$$

where $T_{i i i j}^{ \pm}=T_{(i i i j)} \pm T_{(j j j i)}$, and $S_{i j}=S\left(m_{i}^{2}, m_{j}^{2}\right)$.

Thus, finally (E7) can be expressed as a sum

$$
\begin{aligned}
& \sum_{i, j, k, l} J_{i j k l} T_{i j k l} \\
& =\sum_{i} J_{i} T_{i i i i}+4 \sum_{i<j}\left[\left(J_{i j}+\frac{1}{2} S_{i j}\right) T_{i i i j}^{+}+\frac{3}{2} R_{i j} T_{i i i j}^{-}\right] \\
& \quad+6 \sum_{i<j}\left(J_{i j}-S_{i j}\right) T_{(i i j j)}+12 \sum_{\substack{i \neq j \neq k, j<k}} J_{i i j k} T_{(i i j k)} .
\end{aligned}
$$

\section{APPENDIX F: PROPERTIES OF $\boldsymbol{t}$ COEFFICIENTS}

It is the purpose of this Appendix to establish some important properties of $t$ coefficients which have been used in the computation of the effective action.

We begin from the coefficient $\hat{t}_{i j k}$ given by Eq. (62). For that we need the following expressions:

$$
\begin{gathered}
d_{i j}^{\alpha}=\delta_{i j} \partial^{\alpha}+i \Gamma_{i j}^{\alpha}, \\
\left(d^{2}\right)_{i j}=\sum_{k} d_{i k}^{\alpha} d_{k j}^{\alpha} \\
=\delta_{i j} \partial^{2}+2 i \Gamma_{i j}^{\alpha} \partial^{\alpha}+i\left(\partial^{\alpha} \Gamma_{i j}^{\alpha}\right)-\left(\Gamma^{2}\right)_{i j} .
\end{gathered}
$$

Then for the products we have

$$
\begin{array}{r}
d_{i j}^{\alpha} d_{j k}^{\alpha}\left(d^{2}\right)_{k i}=\delta_{j k}\left(\partial \Gamma_{i j}\right)\left[\left(\partial \Gamma_{k i}\right)+i\left(\Gamma^{2}\right)_{k i}\right] \\
-\Gamma_{i j}^{\alpha} \Gamma_{j k}^{\alpha}\left[i\left(\partial \Gamma_{k i}\right)-\left(\Gamma^{2}\right)_{k i}\right], \\
d_{j k}^{\alpha}\left(d^{2}\right)_{k i} d_{i j}^{\alpha}=\delta_{k i}\left(\partial^{\beta} \Gamma_{j k}^{\alpha}\right)\left(\partial^{\beta} \Gamma_{i j}^{\alpha}\right) \\
+i \Gamma_{k i}^{\beta}\left(\Gamma_{i j}^{\alpha} \partial^{\beta} \Gamma_{j k}^{\alpha}\right)+\Gamma_{i j}^{\alpha} \Gamma_{j k}^{\alpha}\left(\Gamma^{2}\right)_{k i}, \\
\left(d^{2}\right)_{k i} d_{i j}^{\alpha} d_{j k}^{\alpha}= \\
\delta_{i j}\left(\partial \Gamma_{j k}\right)\left[\left(\partial \Gamma_{k i}\right)-i\left(\Gamma^{2}\right)_{k i}\right] \\
+\Gamma_{i j}^{\alpha} \Gamma_{j k}^{\alpha}\left[i\left(\partial \Gamma_{k i}\right)+\left(\Gamma^{2}\right)_{k i}\right],
\end{array}
$$

where a total divergence and open derivatives on the righthand side were omitted. It gives

$$
\begin{aligned}
\hat{t}_{i j k}= & \frac{1}{3}\left[\delta_{i j}\left(\partial \Gamma_{k i}\right)\left(\partial \Gamma_{j k}\right)+\delta_{j k}\left(\partial \Gamma_{i j}\right)\left(\partial \Gamma_{k i}\right)\right. \\
& +\delta_{k i}\left(\partial^{\beta} \Gamma_{j k}^{\alpha}\right)\left(\partial^{\beta} \Gamma_{i j}^{\alpha}\right)+i\left(\delta_{j k} \partial \Gamma_{i j}-\delta_{i j} \partial \Gamma_{j k}\right)\left(\Gamma^{2}\right)_{k i} \\
& \left.+i \Gamma_{k i}^{\beta}\left(\Gamma_{i j}^{\alpha} \stackrel{\leftrightarrow}{\partial^{\beta}} \Gamma_{j k}^{\alpha}\right)\right]+\Gamma_{i j}^{\alpha} \Gamma_{j k}^{\alpha}\left(\Gamma^{2}\right)_{k i} .
\end{aligned}
$$


From this formula, in particular, one finds

$$
\begin{aligned}
3 \hat{t}_{i i j}^{+} & =\hat{t}_{i i j}+\hat{t}_{i j i}+\hat{t}_{j i i}+(i \leftrightarrow j) \\
& =\frac{2}{3}\left(\partial \Gamma_{i j}\right)\left(\partial \Gamma_{j i}\right)+\frac{1}{3}\left(\partial^{\beta} \Gamma_{i j}^{\alpha}\right)\left(\partial^{\beta} \Gamma_{j i}^{\alpha}\right)+\frac{i}{3}\left[F_{i j}^{\alpha \beta} \Gamma_{j i}^{\alpha}\left(\Gamma_{i i}^{\beta}-\Gamma_{j j}^{\beta}\right)+F_{i i}^{\alpha \beta} \Gamma_{i j}^{\alpha} \Gamma_{j i}^{\beta}\right]+\Gamma_{j i}^{\alpha}\left(\Gamma_{i i}^{\alpha}+\Gamma_{j j}^{\alpha}\right)\left(\Gamma^{2}\right)_{i j}+\Gamma_{i j}^{\alpha} \Gamma_{j i}^{\alpha}\left(\Gamma^{2}\right)_{i i}+(i \leftrightarrow j)
\end{aligned}
$$

and

$$
\begin{aligned}
3 \hat{t}_{i i j}^{-} & =\hat{t}_{i i j}+\hat{t}_{i j i}+\hat{t}_{j i i}-(i \leftrightarrow j) \\
& =\frac{2 i}{3}\left(\partial \Gamma_{j i}\right)\left(\Gamma^{2}\right)_{i j}+\frac{i}{3}\left[F_{i j}^{\alpha \beta} \Gamma_{j i}^{\alpha}\left(\Gamma_{i i}^{\beta}+\Gamma_{j j}^{\beta}\right)+F_{i i}^{\alpha \beta} \Gamma_{i j}^{\alpha} \Gamma_{j i}^{\beta}\right]+\Gamma_{j i}^{\alpha}\left(\Gamma_{i i}^{\alpha}-\Gamma_{j j}^{\alpha}\right)\left(\Gamma^{2}\right)_{i j}+\Gamma_{i j}^{\alpha} \Gamma_{j i}^{\alpha}\left(\Gamma^{2}\right)_{i i}-(i \leftrightarrow j) .
\end{aligned}
$$

From (F6) it follows also that in the case of three different masses, $m_{1} \neq m_{2} \neq m_{3}$, the $t$ coefficient $\hat{t}_{(123)}$ is

$$
\hat{t}_{(123)}=\frac{1}{6} \sum_{i \neq j \neq k}\left[\frac{i}{3} \Gamma_{i j}^{\alpha} \Gamma_{j k}^{\beta} F_{k i}^{\alpha \beta}+\Gamma_{i j}^{\alpha} \Gamma_{j k}^{\alpha}\left(\Gamma^{2}\right)_{k i}\right] .
$$

Now consider the coefficient $t_{i j k l}$ given by Eq. (62). Up to a total divergence it can be rewritten as

$$
\begin{aligned}
t_{i j k l}= & \frac{1}{4} \delta_{\alpha \beta \gamma \sigma}\left[4 \Gamma_{i j}^{\alpha} \Gamma_{j k}^{\beta} \Gamma_{k l}^{\gamma} \Gamma_{l i}^{\sigma}+\delta_{j k} \delta_{k l}\left(\partial^{\beta} \Gamma_{i j}^{\alpha}\right)\left(\partial^{\gamma} \Gamma_{l i}^{\sigma}\right)+\delta_{k l} \delta_{l i}\left(\partial^{\gamma} \Gamma_{j k}^{\beta}\right)\left(\partial^{\sigma} \Gamma_{i j}^{\alpha}\right)+\delta_{l i} \delta_{i j}\left(\partial^{\sigma} \Gamma_{k l}^{\gamma}\right)\left(\partial^{\alpha} \Gamma_{j k}^{\beta}\right)+\delta_{i j} \delta_{j k}\left(\partial^{\alpha} \Gamma_{l i}^{\sigma}\right)\left(\partial^{\beta} \Gamma_{k l}^{\gamma}\right)\right. \\
& \left.+i \delta_{k l} \Gamma_{i j}^{\alpha}\left(\Gamma_{l i}^{\sigma} \stackrel{\leftrightarrow}{\partial^{\gamma}} \Gamma_{j k}^{\beta}\right)+i \delta_{j k} \Gamma_{l i}^{\sigma}\left(\Gamma_{k l}^{\gamma} \stackrel{\leftrightarrow}{\partial^{\beta}} \Gamma_{i j}^{\alpha}\right)+i \delta_{l i} \Gamma_{j k}^{\beta}\left(\Gamma_{i j}^{\alpha} \stackrel{\leftrightarrow}{\partial^{\sigma}} \Gamma_{k l}^{\gamma}\right)+i \delta_{i j} \Gamma_{k l}^{\gamma}\left(\Gamma_{j k}^{\beta} \stackrel{\leftrightarrow}{\partial^{\alpha}} \Gamma_{l i}^{\sigma}\right)\right] .
\end{aligned}
$$

Hence, we have

$$
t_{i i i i}=2\left(\partial \Gamma_{i i}\right)^{2}+\left(\partial^{\alpha} \Gamma_{i i}^{\beta}\right)^{2}+3\left(\Gamma_{i i}^{\alpha} \Gamma_{i i}^{\alpha}\right)^{2}
$$

Further, from the definition of the coefficients and the property of the cyclic permutation, it follows that

$$
\begin{gathered}
t_{i i i j}=t_{i i j i}=t_{i j i i}=t_{j i i i}=t_{(i i i j)}, \\
t_{i i j j}=t_{i j j i}=t_{j j i i}=t_{j i i j}, \\
t_{i j i j}=t_{j i j i} .
\end{gathered}
$$

Therefore,

$$
\begin{aligned}
t_{(i i i j)} & =\frac{1}{4} \delta_{\alpha \beta \gamma \sigma}\left[\left(\partial^{\alpha} \Gamma_{j i}^{\sigma}\right)\left(\partial^{\beta} \Gamma_{i j}^{\gamma}\right)+4 \Gamma_{i i}^{\alpha} \Gamma_{i i}^{\beta} \Gamma_{i j}^{\gamma} \Gamma_{j i}^{\sigma}+i\left(\partial^{\alpha} \Gamma_{j i}^{\sigma}\right) \Gamma_{i i}^{\beta} \Gamma_{i j}^{\gamma}-i\left(\partial^{\beta} \Gamma_{i j}^{\gamma}\right) \Gamma_{j i}^{\sigma} \Gamma_{i i}^{\alpha}\right] \\
& =\frac{1}{4}\left\{2\left(\partial \Gamma_{i j}\right)\left(\partial \Gamma_{j i}\right)+\left(\partial^{\alpha} \Gamma_{i j}^{\beta}\right)\left(\partial^{\alpha} \Gamma_{j i}^{\beta}\right)+i \Gamma_{i i}^{\beta}\left[\left(\Gamma_{i j}^{\beta} \stackrel{\leftrightarrow}{\partial^{\alpha}} \Gamma_{j i}^{\alpha}\right)-\left(\Gamma_{j i}^{\beta} \stackrel{\leftrightarrow}{\partial^{\alpha}} \Gamma_{i j}^{\alpha}\right)+\left(\Gamma_{i j}^{\alpha} \stackrel{\leftrightarrow}{\partial^{\beta}} \Gamma_{j i}^{\alpha}\right)\right]\right\}+\left(\Gamma_{i i} \Gamma_{i i}\right)\left(\Gamma_{i j} \Gamma_{j i}\right)+2\left(\Gamma_{i i} \Gamma_{i j}\right)\left(\Gamma_{i i} \Gamma_{j i}\right)
\end{aligned}
$$

From (F15), we obtain

$$
\begin{aligned}
t_{i i i j}^{ \pm}= & \delta_{ \pm+}\left[\left(\partial \Gamma_{i j}\right)\left(\partial \Gamma_{j i}\right)+\frac{1}{2}\left(\partial^{\alpha} \Gamma_{i j}^{\beta}\right)\left(\partial^{\alpha} \Gamma_{j i}^{\beta}\right)\right]+\frac{i}{4}\left(\Gamma_{i i}^{\beta} \mp \Gamma_{j j}^{\beta}\right)\left[\left(\Gamma_{i j}^{\beta} \stackrel{\leftrightarrow}{\partial^{\alpha}} \Gamma_{j i}^{\alpha}\right)-\left(\Gamma_{j i}^{\beta} \stackrel{\leftrightarrow}{\partial^{\alpha}} \Gamma_{i j}^{\alpha}\right)+\left(\Gamma_{i j}^{\alpha} \stackrel{\leftrightarrow}{\partial^{\beta}} \Gamma_{j i}^{\alpha}\right)\right] \\
& +\left[\left(\Gamma_{i i} \Gamma_{i i}\right)\left(\Gamma_{i j} \Gamma_{j i}\right)+2\left(\Gamma_{i i} \Gamma_{i j}\right)\left(\Gamma_{i i} \Gamma_{j i}\right) \pm(i \leftrightarrow j)\right],
\end{aligned}
$$

where $\delta_{++}=1$, and $\delta_{-+}=0$. 
Next, we calculate the coefficient $t_{(i i j j)}$. Using the formulas (E8), (F13), and (F14) it is easy to establish that

$$
t_{(i i j j)}=\frac{1}{3}\left(2 t_{i i j j}+t_{i j i j}\right) \text {. }
$$

The formula (F10) gives now

$$
\begin{aligned}
t_{(i i j j)}= & \frac{1}{3}\left\{\left(\Gamma_{i i} \Gamma_{i j}\right)\left(\Gamma_{j j} \Gamma_{j i}\right)+\left(\Gamma_{i j} \Gamma_{j j}\right)\left(\Gamma_{j i} \Gamma_{i i}\right)+\left(\Gamma_{i i} \Gamma_{j j}\right)\left(\Gamma_{i j} \Gamma_{j i}\right)+\left(\Gamma_{i j} \Gamma_{j i}\right)^{2}+\frac{1}{2}\left(\Gamma_{i j} \Gamma_{i j}\right)\left(\Gamma_{j i} \Gamma_{j i}\right)\right. \\
& \left.+\frac{i}{2} \Gamma_{i i}^{\alpha}\left[\left(\Gamma_{j i}^{\alpha} \stackrel{\leftrightarrow}{\partial^{\beta}} \Gamma_{i j}^{\beta}\right)+\left(\Gamma_{j i}^{\beta} \stackrel{\leftrightarrow}{\partial^{\alpha}} \Gamma_{i j}^{\beta}\right)+\left(\Gamma_{j i}^{\beta} \stackrel{\leftrightarrow}{\partial^{\beta}} \Gamma_{i j}^{\alpha}\right)\right]+(i \leftrightarrow j)\right\} .
\end{aligned}
$$

Finally, consider the coefficient $t_{(i i j k)}$. In accord with the definition (E8) and the cyclic property (62) we have

$$
\begin{aligned}
t_{(i i j k)} & =\frac{1}{3}\left(t_{i i j k}+t_{i i k j}+t_{i k i j}\right) \\
& =\frac{1}{3} \delta_{\alpha \beta \gamma \sigma}\left[\Gamma_{i i}^{\alpha} \Gamma_{i j}^{\beta} \Gamma_{j k}^{\gamma} \Gamma_{k i}^{\sigma}+\Gamma_{i i}^{\alpha} \Gamma_{i k}^{\beta} \Gamma_{k j}^{\gamma} \Gamma_{j i}^{\sigma}+\Gamma_{i j}^{\alpha} \Gamma_{j i}^{\beta} \Gamma_{i k}^{\gamma} \Gamma_{k i}^{\sigma}+\frac{i}{4} \Gamma_{j k}^{\gamma}\left(\Gamma_{i j}^{\beta} \overleftrightarrow{\partial}^{\alpha} \Gamma_{k i}^{\sigma}\right)+\frac{i}{4} \Gamma_{k j}^{\gamma}\left(\Gamma_{i k}^{\beta} \stackrel{\leftrightarrow}{\partial^{\alpha}} \Gamma_{j i}^{\sigma}\right)\right] .
\end{aligned}
$$

In conclusion, we recall that the coefficients depending on $Y: t_{i j}$ and $t_{i j k}$ were calculated in the main part of the work.

[1] J. Hadamard, Le problème de Cauchy et les équations aux dérivées partielles linéaires hyperboliques (Hermann et Cie, Paris, 1932).

[2] V. A. Fock, Proper time in classical and quantum mechanics, Izv. Akad. Nauk. USSR (Phys.) 4-5, 551 (1937).

[3] V. A. Fock, Die eigenzeit in der klassischen und in der quantenmechanik, Phys. Zs. Sowjet. 12, 404 (1937).

[4] Y. Nambu, The use of the proper time in quantum electrodynamics I, Prog. Theor. Phys. 5, 82 (1950).

[5] J. Schwinger, On gauge invariance and vacuum polarization, Phys. Rev. 82, 664 (1951).

[6] B. S. DeWitt, Dynamical Theory of Groups and Fields (Gordon \& Breach, New York, 1965).

[7] B.S. DeWitt, Quantum field theory in curved spacetime, Phys. Rep. 19, 295 (1975).

[8] A. O. Barvinsky and G. A. Vilkovisky, Beyond the Schwinger-DeWitt technique: Converting loops into trees and in-in currents, Nucl. Phys. B282, 163 (1987).

[9] A. O. Barvinsky and G. A. Vilkovisky, Covariant perturbation theory (II). Second order in the curvature. General algorithms, Nucl. Phys. B333, 471 (1990).

[10] I. G. Avramidi, A covariant technique for the calculation of the one-loop effective action, Nucl. Phys. B355, 712 (1991).

[11] I. G. Avramidi, Heat Kernel and Quantum Gravity, in Lecture Notes in Physics Monographs Vol. 64 (Springer, Berlin, Heidelberg, 2000), https://doi.org/10.1007/3-54046523-5.

[12] A. Dhar, R. Shankar, and S. R. Wadia, Nambu-JonaLasinio-type effective Lagrangian: Anomalies and nonlinear
Lagrangian of low-energy, large- $N$ QCD, Phys. Rev. D 31, 3256 (1985).

[13] D. Ebert and H. Reinhardt, Effective chiral hadron Lagrangian with anomalies and Skyrme terms from quark flavour dynamics, Nucl. Phys. B271, 188 (1986).

[14] R. D. Ball, Chiral gauge theory, Phys. Rep. 182, 1 (1989).

[15] C. Callan and F. Wilczek, On geometric entropy, Phys. Lett. B 333, 55 (1994).

[16] M. Bordag, U. Mohideen, and V. M. Mostepanenko, New developments in the Casimir effect, Phys. Rep. 353, 1 (2001).

[17] D. V. Vassilevich, Heat kernel expansion: User's manual, Phys. Rep. 388, 279 (2003).

[18] Y. Nambu and G. Jona-Lasinio, Dynamical model of elementary particles based on an analogy with superconductivity. I, Phys. Rev. 122, 345 (1961).

[19] Y. Nambu and G. Jona-Lasinio, Dynamical model of elementary particles based on an analogy with superconductivity. II, Phys. Rev. 124, 246 (1961).

[20] M. K. Volkov, Meson Lagrangians in a superconductor quark model, Ann. Phys. (N.Y.) 157, 282 (1984).

[21] G. Passarino, XEFT, the challenging path up the hill: $\operatorname{dim}=$ 6 and $\operatorname{dim}=8$, arxiv:1901.04177.

[22] C. Lee, H. Min, and P. Y. Pac, Heavy fermions in gauge theories, Nucl. Phys. B202, 336 (1982).

[23] C. Lee, T. Lee, and H. Min, Background-field and Schwinger-DeWitt proper-time algorithm for the lowenergy effective-field-theory action, Phys. Rev. D 39, 1681 (1989). 
[24] C. Lee, T. Lee, and H. Min, Generalized Schwinger-DeWitt expansions and effective field theories, Phys. Rev. D 39, 1701 (1989).

[25] A. A Osipov, Fock-Schwinger method in the case of different masses, JETP Lett. 113, 413 (2021).

[26] A. A Osipov, Proper-time method for unequal masses, Phys. Lett. B 817, 136300 (2021).

[27] A. A. Osipov and B. Hiller, Generalized proper-time approach for the case of broken isospin symmetry, Phys. Rev. D 63, 094009 (2001).

[28] A. A. Osipov and B. Hiller, Large mass invariant asymptotics of the effective action, Phys. Rev. D 64, 087701 (2001).

[29] A. A. Osipov and B. Hiller, Inverse mass expansion of the one-loop effective action, Phys. Lett. B 515, 458 (2001).

[30] T. Alanne, N. Bizot, G. Cacciapaglia, and F. Sannino, Classification of NLO operators for composite Higgs models, Phys. Rev. D 97, 075028 (2018).

[31] G. C. Branco, P. M. Ferreira, L. Lavoura, M. N. Rebelo, M. Sherd, and J. P. Silva, Theory and phenomenology of two-Higgs-doublet models, Phys. Rep. 516, 1 (2012).

[32] L. Bian, N. Chen, W. Su, Y. Wu, and Y. Zhang, Future prospects of mass-degenerate Higgs bosons in the $C P$ conserving two-Higgs-doublet model, Phys. Rev. D 97, 115007 (2018).

[33] H. E. Haber, O. M. Ogreid, P. Osland, and M. N. Rebelo, Symmetries and mass degeneracies in the scalar sector, J. High Energy Phys. 01 (2019) 042.
[34] R. Herrera-Siklódy, J. I. Latorre, P. Pascual, and J. Taron, Chiral effective lagrangian in the large- $N_{c}$ limit: The nonet case, Nucl. Phys. B497, 345 (1997).

[35] A. A. Osipov, B. Hiller, and A. H. Blin, Effective multiquark interactions with explicit breaking of chiral symmetry, Phys. Rev. D 88, 054032 (2013).

[36] E. Witten, Cosmic separation of phases, Phys. Rev. D 30, 272 (1984).

[37] J. Moreira, J. Morais, B. Hiller, A. A. Osipov, and A. H. Blin, Strange quark matter in the presence of explicit symmetry breaking interactions, Phys. Rev. D 91, 116003 (2015).

[38] M. N. Chernodub, Spontaneous Electromagnetic Superconductivity of Vacuum in a Strong Magnetic Field: Evidence from the Nambu-Jona-Lasinio Model, Phys. Rev. Lett. 106, 142003 (2011).

[39] R. Gatto and M. Ruggieri, Deconfinement and chiral symmetry restoration in a strong magnetic background, Phys. Rev. D 83, 034016 (2011).

[40] M. K. Volkov, Low energy physics of mesons in the superconducting quark model, Phys. Elem. Part. At. Nucl. 17, 433 (1986), http://www1.jinr.ru/Archive/Pepan/1986v17/v-17-3/2.htm.

[41] G. P. H. Styan, Hadamard products and multivariate statistical Analysis, Linear Algebra Appl. 6, 217 (1973).

[42] J. Morais, B. Hiller, and A. A. Osipov, A general framework to diagonalize vector-scalar and axial-vector-pseudoscalar transitions in the effective meson Lagrangian, Phys. Lett. B 773, 277 (2017). 